International Journal of Bifurcation and Chaos, Vol. 14, No. 2 (2004) 551-584

(c) World Scientific Publishing Company

\title{
RECEPTIVE FIELD ATLAS AND RELATED CNN MODELS
}

\author{
V. GÁL, J. HÁMORI, T. ROSKA, D. BÁLYA, ZS. BOROSTYÁNKÖI, M. BRENDEL, \\ K. LOTZ, L. NÉGYESSY, L. ORZÓ, I. PETRÁS, CS. REKECZKY, J. TAKÁCS, \\ P. VENETIÁNER, Z. VIDNYÁNSZKY and Á. ZARÁNDY \\ Analogical and Neural Computing Laboratory, \\ Hungarian Academy of Sciences Computer and Automation Research Institute, \\ Lágymányosi u. 13, Budapest, H-1111, Hungary
}

Received January 27, 2003; Revised October 3, 2003

\begin{abstract}
In this paper we demonstrate the potential of the cellular nonlinear/neural network paradigm (CNN) that of the analogic cellular computer architecture (called CNN Universal Machine CNN-UM) in modeling different parts and aspects of the nervous system. The structure of the living sensory systems and the CNN share a lot of features in common: local interconnections ("receptive field architecture"), nonlinear and delayed synapses for the processing tasks, the potentiality of feedback and using the advantages of both the analog and logic signal-processing mode. The results of more than ten years of cooperative work of many engineers and neurobiologists have been collected in an atlas: what we present here is a kind of selection from these studies emphasizing the flexibility of the CNN computing: visual, tactile and auditory modalities are concerned.
\end{abstract}

Keywords: Receptive fields; receptive field calculus; CNN; cellular neural networks; cellular nonlinear networks; local interconnections; visual, auditory and tactile system modeling; feedforward; feedback network layers.

\section{Introduction}

The core idea behind our work is that the architecture of different functional levels in the sensory system has an invariant concept: the parallelorganized 2D grids of processing and sensing elements (sensors, neurons or cells) make up a threedimensional array having very dense short-distance, neighborhood connections and a relatively small number of remote synapses.

In this context the term "receptive field organization" briefly denoted here as receptive field (RF), can be defined as a limited region of a layer in which stimulation leads to response of a particular sensory neuron/cell in another layer. In addition, we include the actual interaction synaptic weight pattern of the dendritic (feed forward) and recurrent (feedback) synapses. Apart from the exact position, cells within a layer have similar, if not identical, receptive field properties, which opens a door for engineers to create physically implementable models of the sensory networks.

The architecture described by the CNN cellular neural/nonlinear network paradigm [Chua \& Yang, 1988; Chua \& Roska, 1993] proved to be an excellent host for these models: the numerous analogies between the biological systems and the generic structure of the CNN enables us to develop CNN models based on a few "receptive field interaction prototypes".

Hence our Receptive Field Atlas is a catalog for several sensory examples, however, it demonstrates the role of the prototype "CNN computer" to be programmed to behave as different organs. Since 1990, our collaborative groups have been working on modeling the visual, auditory and somatosensory pathways. This multidisciplinary 


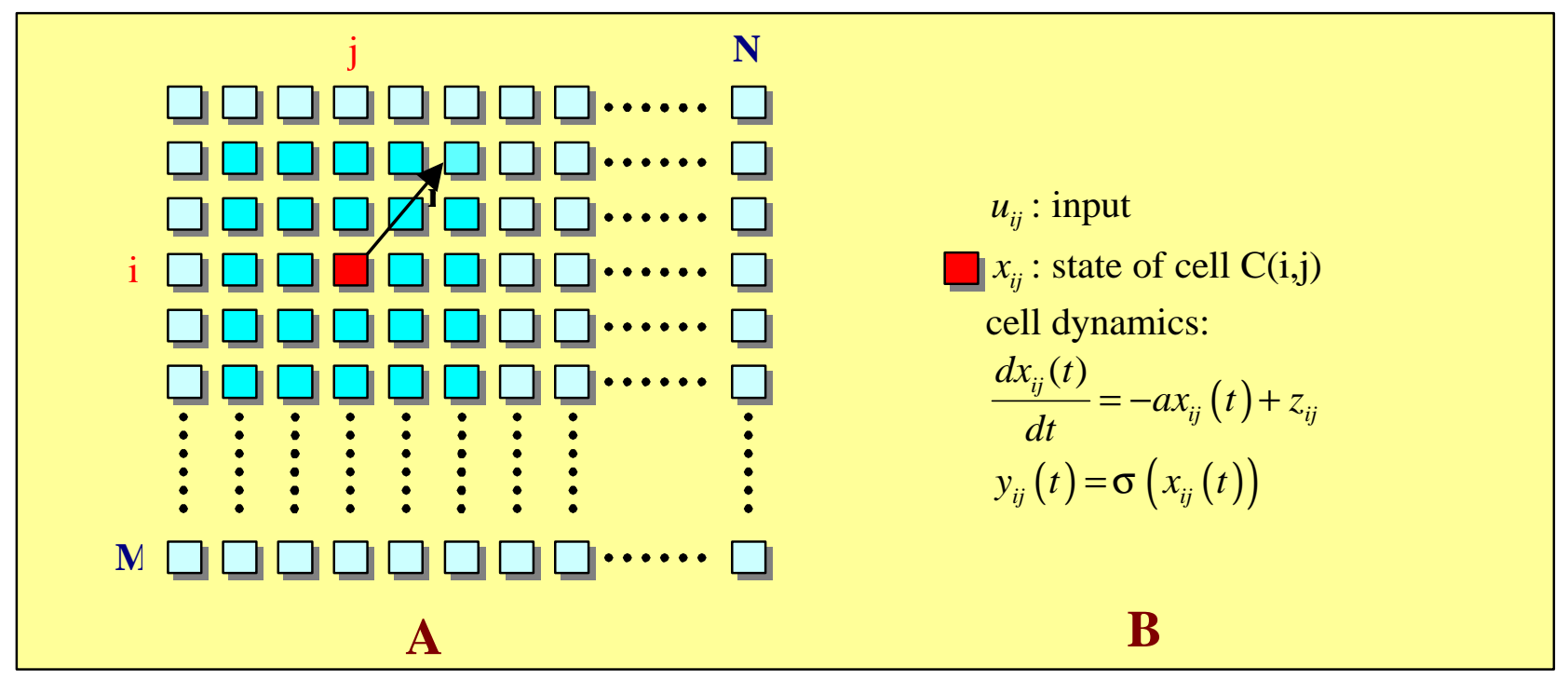

Fig. 1. The CNN consists of a topographic one-, two- or three-dimensional array of cells. A two-dimensional layer (without inter-cell connection symbols) is shown in (A). One simple isolated cell dynamics is described in (B). The local interaction or sphere of influence is denoted by $S_{r}(i j)$ and its radius is indicated by the black arrow. Hence, $3 \times 3,5 \times 5,7 \times 7$ neighborhoods are the typical receptive field sizes.

research of our laboratories, engineers and physicists in information technology, as well as medical doctors and neurobiologists in brain research was a challenge for all of us. Fortunately, we found the powerful modeling paradigm (the $\mathrm{CNN}$ ) and dedicated researchers as excellent cooperating partners.

This paper provides an insight into our results through a number of examples without presenting the whole paths that led us to the fine-tuned models. It shows that many functionalities we studied could be modeled in a neuromorpic way, by a receptive field calculus, defined on the analogic cellular computer architecture (called CNN Universal Machine - CNN-UM) [Roska \& Chua, 1993a]. The details of retinal modeling are described in another paper of this issue.

This way, all the results are directly applicable in artificial visual, tactile and auditory system, and in various forms they can be implemented in topographic microprocessors (based on the CNN-UM).

During the twelve years, we have also learned how many questions are still waiting for answers. In addition to the modeling results, we have developed a modeling tool called REFINE-C as a special version of the CANDY simulator. ${ }^{1}$

A special simple version of a single-layer $\mathrm{CNN}$ is shown in Fig. 1 and described by the state and output equations:

$$
\begin{aligned}
\tau \frac{d x_{i j}(t)}{d t}= & -a x_{i j}+\sum_{k l \in S_{r}(i j)} A_{i j k l} y_{k l}(t) \\
& +\sum_{k l \in S_{r}(i j)} B_{i j k l} u_{k l}(t)+z_{i j} \\
y_{i j}(t)= & \sigma\left(x_{i j}(t)\right) \\
i= & 1,2, \ldots, M, \quad j=1,2, \ldots, N
\end{aligned}
$$

where the two sums in Eq. (1a) represent the incoming interactions from the cell outputs (A) and inputs (B) of the neighboring cells in the sphere of influence $S_{r}(i j)$, respectively. Considering a multidimensional $(>2)$ CNN these connections can arise from different layers. In many cases $z_{i j}$ - a bias term (or threshold) - is a space invariant scalar: $z_{i j}=z$. The time constant $\tau$ is also a single value characterizing all the cells within a layer.

Hence, a cloning template which completely determines the properties of the CNN $C:\{A, B, z\}$, describing the simplest interaction weight pattern for $r=1$ is:

$$
A=\left[\begin{array}{lll}
a_{-1-1} & a_{10} & a_{-11} \\
a_{0-1} & a_{00} & a_{01} \\
a_{1-1} & a_{10} & a_{11}
\end{array}\right]
$$




$$
B=\left[\begin{array}{lll}
b_{-1-1} & b_{10} & b_{-11} \\
b_{0-1} & b_{00} & b_{01} \\
b_{1-1} & b_{10} & b_{11}
\end{array}\right] \quad z=\left[z_{0}\right]
$$

For example an off-center on-surround recursive (A) receptive field and an on-center off surround dendritic (B) receptive field have the following templates.

$$
A=\left[\begin{array}{lll}
+ & + & + \\
+ & - & + \\
+ & + & +
\end{array}\right] \quad B=\left[\begin{array}{lll}
- & - & - \\
- & + & - \\
- & - & -
\end{array}\right]
$$

The threshold value is $z$. There are nonlinear and delay type templates as well, representing nonlinear or delayed synapse characteristics: $\hat{A}(i j ; k l)\left(x_{k l} u_{k l}\right), \hat{B}(i j ; k l)\left(u_{k l}\right)$ and $A^{\tau}(i j ; k l) y_{k l}(t-$ $\tau), B^{\tau}(i j ; k l) u_{k l}(t-\tau)$.

More details (with multilayer examples) can also be found in the undergraduate textbook [Chua \& Roska, 2002].

\section{Typical Single-Neuron and Synapse Models}

In this chapter we provide an overview of singleneuron models favored by neuroscientist and make a comparison with the typical elementary units in CNN architectures. In order to review the relevant terms used by biologists, a semantic description is given.

In general, a nerve cell can be divided into three main parts based on functional and morphological characteristics (see Fig. 2):

- Dendrites: accommodate synapses, the "inputinterfaces" of neurons. Dendrites feed stimulus coming from other cells towards the soma. The adjustment of the weight of connections between neurons occurs mainly here.

- Soma: Receives and integrates stimuli from other cells, provides a converging pathway for the electrotonic signals arising in the dendrites.

- Axon (and axon-hillock): In addition to a threshold operation, the axon-hillock converts the signals in similar manner as the frequency modulators; it transmits the integrated and converted signals over long distances. It also provides output interface towards other cells.

A key step to having a good engineering model is to understand the electrochemical forces and reactions taking place in a cell. After examining the

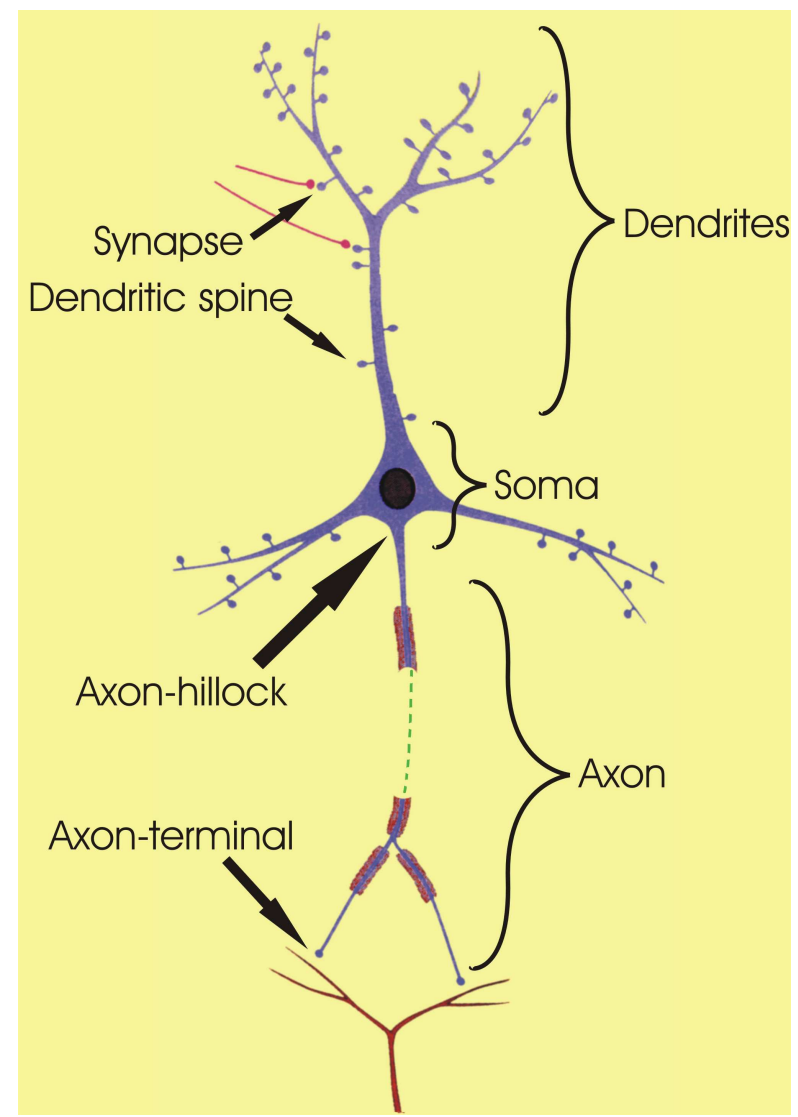

Fig. 2. The neuron.

distribution of the different ions inside (intracellular space) and outside (extracellular space) the neuron, their flow along the cell's axis and through the numerous special channels integrated in the isolating cell membrane one can design an "equivalent electrical circuit" representing the living neuron (see Fig. 3). Choosing appropriate circuit elements the model faithfully reproduces the most important components of the dynamics of neurons [Johnston \& Wu, 1995]. In Table 1 we can find the corresponding terms from biology and engineering describing analogous models of the neuron.

Here we are going to discuss a very simple "twocompartment" model, where two state equations describe the behavior of the soma/dendrite and the axon part represented by two attached electrical circuits (see Fig. 3):

$$
\begin{aligned}
C_{m} \frac{d V_{\text {soma }}}{d t}= & g_{\mathrm{K}}\left(V_{\text {soma }}-E_{\mathrm{k}}\right)+g_{\mathrm{Na}}\left(V_{\text {soma }}-E_{\mathrm{Na}}\right) \\
& +g_{\mathrm{Cl}}\left(V_{\text {soma }}-E_{\mathrm{Cl}}\right) \\
& +g_{\text {syn }}\left(V_{\text {soma }}-E_{\text {syn }}\right) \\
& +\frac{V_{\text {axon }}-V_{\text {soma }}}{R_{\mathrm{ax}}}
\end{aligned}
$$


Table 1. Corresponding expressions in neurobiological and engineering models.

\begin{tabular}{|c|c|c|}
\hline \multicolumn{2}{|l|}{ Biological terms } & $\begin{array}{l}\text { Electric circuit } \\
\text { equivalent }\end{array}$ \\
\hline \multicolumn{2}{|c|}{$\begin{array}{l}\text { Membrane capacitance - the dielectric property of the lipid part of the } \\
\text { membrane (almost constant in space and time) }\end{array}$} & Capacitor \\
\hline \multirow{2}{*}{$\begin{array}{l}\text { Membrane resistance - the resistance of a certain } \\
\text { patch of a membrane depends on the conductance of } \\
\text { the different channel types and their local } \\
\text { concentrations }\end{array}$} & $\begin{array}{l}\text { Channels with } \\
\text { changing } \\
\text { conductance }\end{array}$ & $\begin{array}{l}\text { Voltage controlled } \\
\text { resistor, or current } \\
\text { source(VCCS)etc. }\end{array}$ \\
\hline & $\begin{array}{l}\text { Passive } \\
\text { channels }\end{array}$ & Ohmic resistor \\
\hline \multicolumn{3}{|l|}{ Core (axial) resistance } \\
\hline \multicolumn{2}{|l|}{ Equilibrium potential of individual ions } & Voltage source \\
\hline
\end{tabular}

$$
\begin{aligned}
C_{m} \frac{d V_{\text {axon }}}{d t}= & g_{\mathrm{K}}\left(V_{\text {axon }}-E_{\mathrm{k}}\right) \\
& +g_{\mathrm{Na}}\left(V_{\text {axon }}-E_{\mathrm{Na}}\right)+g_{\mathrm{Cl}}\left(V_{\text {axon }}-E_{\mathrm{Cl}}\right) \\
& +\frac{V_{\text {soma }}-V_{\text {axon }}}{R_{\mathrm{ax}}}
\end{aligned}
$$

where $g_{\mathrm{Na}}, g_{\mathrm{K}}, g_{\mathrm{Cl}}$ : conductance of the membrane for $\mathrm{Na}, \mathrm{K}, \mathrm{Cl}$ ions, respectively. $g_{\mathrm{syn}}$ is a special conductance whose actual value is defined by the activity of presynaptic (connecting) neurons. $E_{\mathrm{Na}}$, $E_{\mathrm{K}}, E_{\mathrm{Cl}}$ are equilibrium potentials (voltage sources) of the individual ions in connection with their unequal distribution between the extra- and intracellular space. $C_{m}$ is the membrane capacitance, $V_{\text {soma }}$ and $V_{\text {axon }}$ are the transmembrane potentials in the axon and the soma compartment. The "axial" resistor connecting the two units is denoted by $R_{\mathrm{ax}}$.

These kinds of models have major significance for two reasons: they are probably the most popular among neuroscientists, and what is more important for us, the elementary units of CNN architectures described in the Receptive Field Atlas can be interpreted as two compartment neurons (compare Figs. 3 and 4).

We did not mention the so-called "spiking" phenomenon of neurons in detail: cells can respond to constant stimuli with repeating abrupt changes action potentials - in their transmembrane voltage. Unique voltage controlled conductances play a crucial role here: the resistance of these ion channels depends highly on the actual membrane voltage.

In the CNN world this is implemented via voltage controlled current sources (VCCS). A VCCS template defines the current flowing through the modeled channel:

$$
I_{x y}^{\mathrm{VCC}}(i, j ; k, l)=C_{i, j ; k, l} \cdot g\left(\nu_{y k l}^{\text {source }}\right)\left(E_{r}-\nu_{x i j}\right)
$$

where $C$ is a matrix of linear coefficients, $g$ is the conductance function dependent on the output voltage of unit $(k, l)$ in the neighborhood of $(i, j)$ on the source layer, and $E_{r}$ is the value of the series voltage source (reversal or equilibrium potential).

\subsection{Synaptic mechanisms}

The communication between neurons is "implemented" via synapses, which mean connections between axon-terminals and dendrites in general. The activation of presynaptic cells triggers the release of so-called neurotransmitters in the axon terminal. There are special ligand gated channels "ionotrop receptors" - integrated in the membrane of the dendrite which bind the transmitters and can change their conductance to specific ions accordingly. The result is either an elevation or a drop in the membrane voltage, which means excitatory or inhibitory coupling, respectively.

In addition to this relatively slow way of communication - the interval is further extended by the long travel of signals along the cell's processes - neuroscientists found direct electrical connections ("gap junctions"). These allow electrical coupling between two cells so an action potential in one cell moves directly into the other, without the $0.4-$ $1 \mathrm{~ms}$ delay observed in chemical synapses. Table 2 provides an overview of the types of "synapses" or "templates" used in our Receptive Field models. 


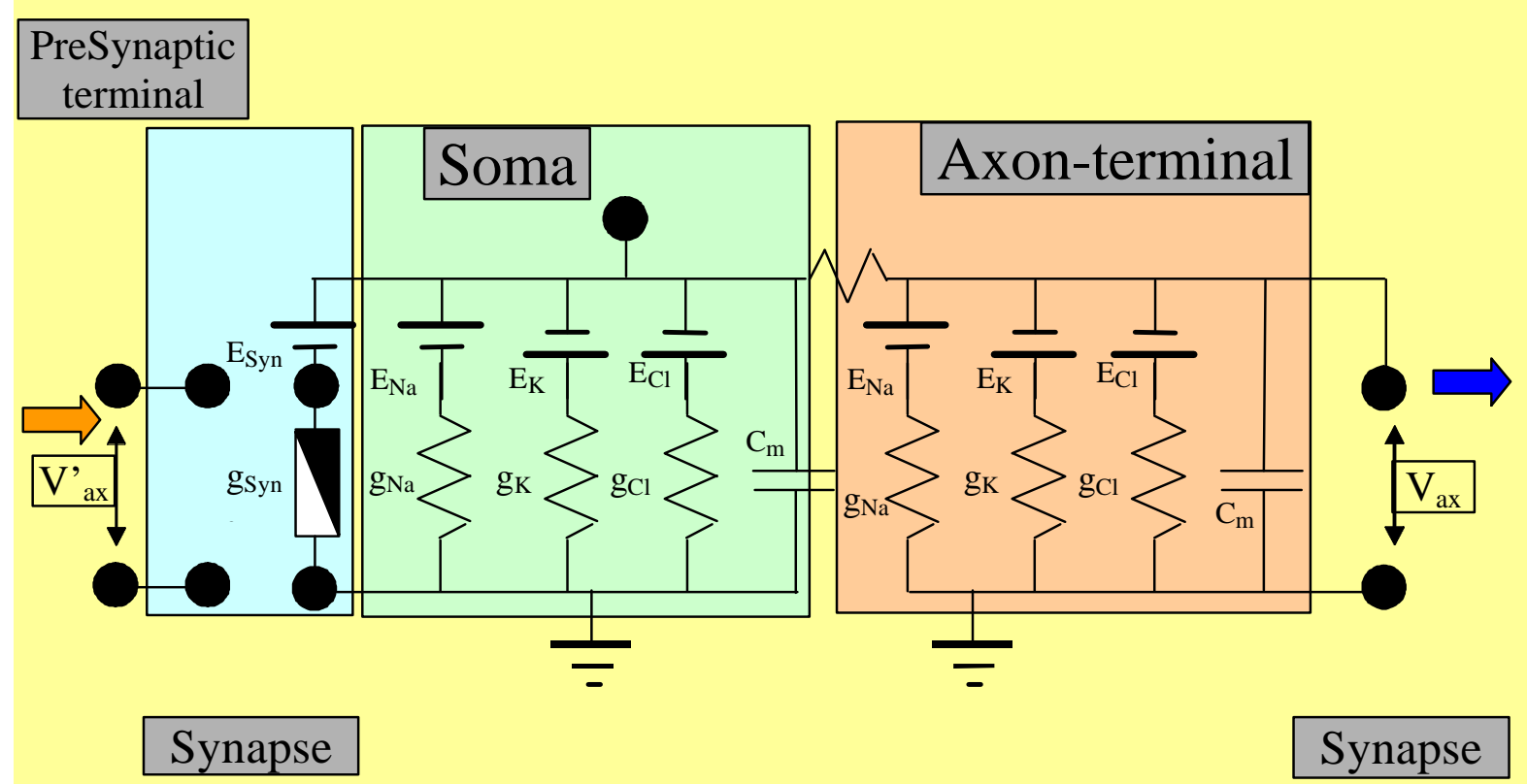

Fig. 3. Simplified two-compartmental model of a simple neuron. $g_{\text {syn }}$ represents the synaptic conductance, which is a nonlinear function of $V_{\mathrm{ax}}^{\prime}$, the transmembrane potential at the presynaptic axon terminal (of the presynaptic cell). The source of input and the direction of output signals are denoted by orange and blue colored arrows, respectively.

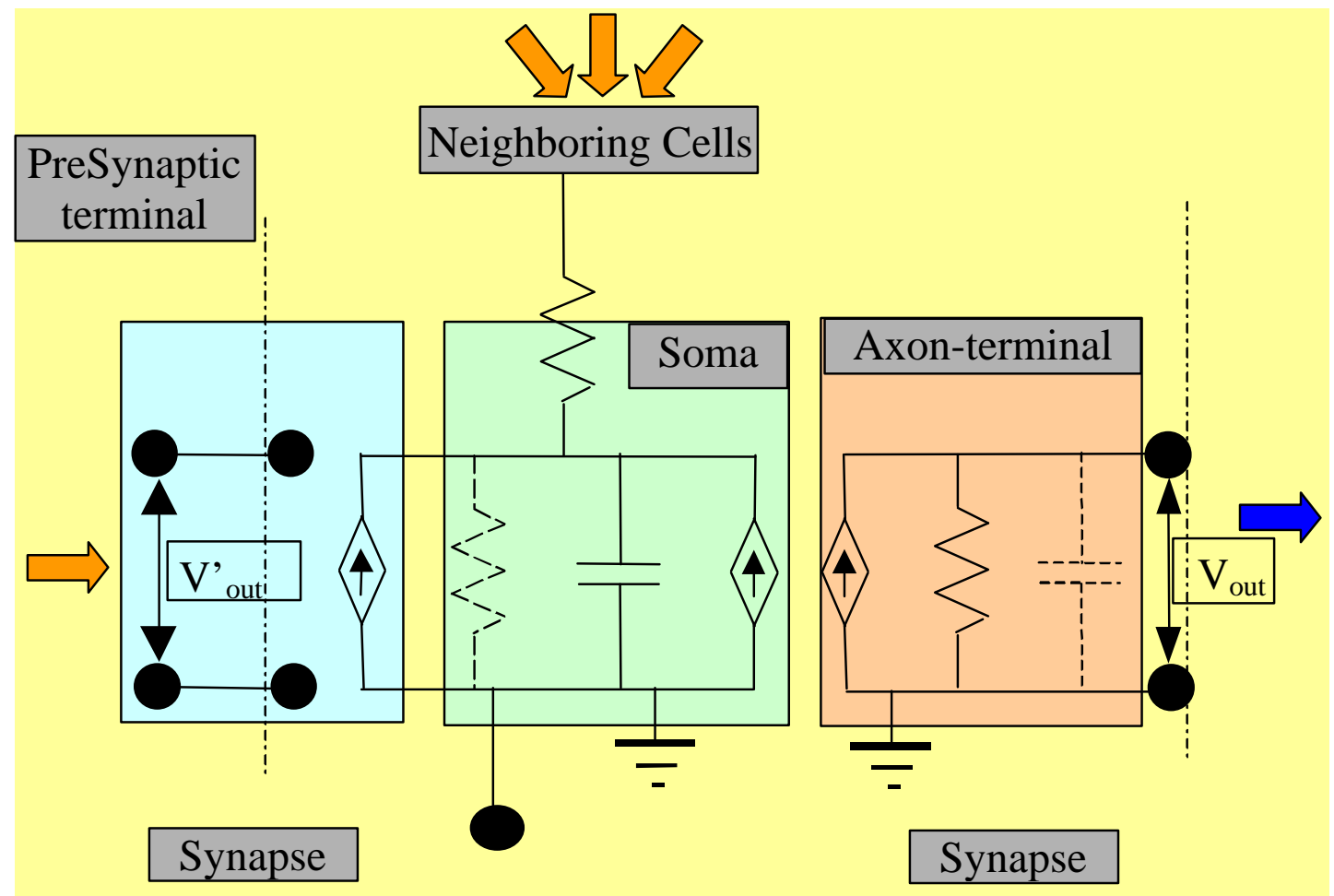

Fig. 4. Basic CNN cell with "neurobiological labels". $V_{\text {out }}^{\prime}$ is the output potential of the "presynaptic cell" (e.g. from another layer), $V_{\text {out }}$ is the output voltage of the cell displayed here. The source of input and the direction of output signals are denoted by orange and blue colored arrows, respectively. 
Table 2. Different types of synapses.
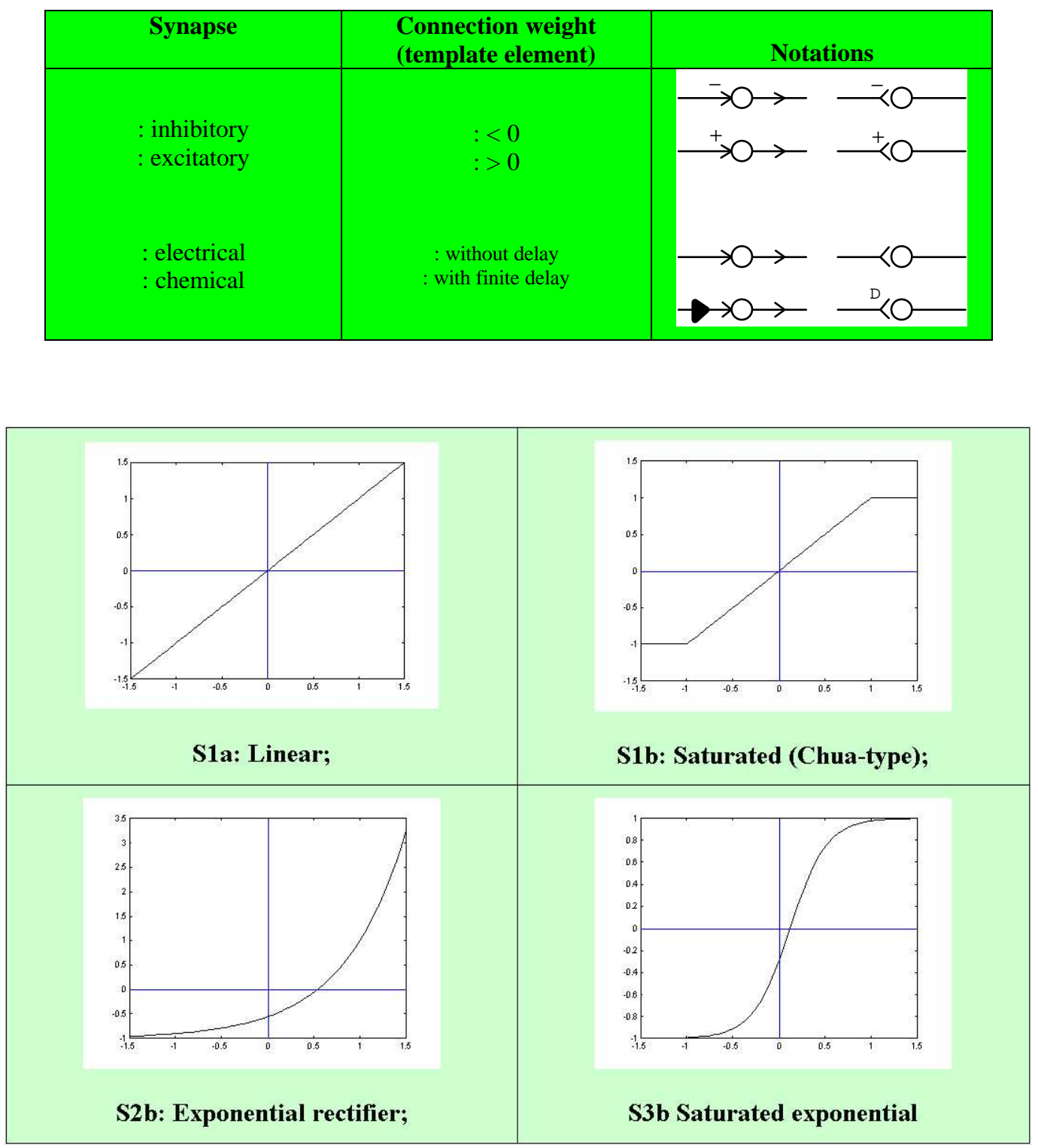

Fig. 5. Characteristics of different synapse prototypes. In the graphs S2b and S3b the curves do not pass through the origin but are shifted towards the positive direction representing real neuronal characteristics.

Finally we give a rough comparison between the models of silicon and nerve cells.

Similarities and analogies:

- The state of a cell (somatic potential = potential at the core) represents either transmembrane voltage or action potential frequency.

- Action potentials are generated neither in CNN nor in neurons, unless they contain voltage sensitive unit (VCCS template or channel).

- We can find ohmic resistor(s) (membrane resistance) and capacitor(s) (membrane capacity) in both systems' core subunit.
- The outputs of the systems are nonlinear functions of the core voltages.

- The inputs of a unit are nonlinear functions of the output voltage of the presynaptic cells.

- One side (the ground or extracellular side) of each unit in a network can be treated as equipotential surface.

\section{Differences:}

- Electrochemical forces are not represented separately, since CNN does not distinguish between different kinds of charged particles. 
- Unlike neurons, the CNN cells (of different layers) influence each other via a voltage dependent current generator. The value of the input current depends on the output voltage of the "presynaptic" cell. In biological systems the transmembrane potential at axon-terminals has an indirect effect on the synaptic conductance only. Thus, the input current also depends on the actual potential (state) of the postsynaptic cell.

\section{Modeling with Receptive Field Interaction Prototypes (Multilayer CNN as a Prototype Description)}

As we explained in the introduction part of the paper, the neuromorphical CNN models are based on a few "receptive field interaction prototypes".

The following prototypes are considered to be necessary:

- Synapse prototypes $(S i)$

- Receptive field prototypes $(R f)$

- Layer prototypes $(\mathrm{Li})$

These prototypes can be defined and preprogrammed via their few controllable parameters, and constitute the Receptive Field Interaction prototypes (RFIi).

The applied synapse prototypes can be: $S 1 a$ : Linear; $S 1 b$ : Saturated; $S 2 a$ : Linear rectifier; $S 2 b$ : Exponential rectifier; $S 3 a$ : Custom exponential; $S 3 b$ : Sigmoid exponential.

A couple of the possible synaptic prototypes are shown in Fig. 5.

The receptive field prototypes:

We can define the receptive field prototypes given by the pattern of weights in the representative template.

For example, a symmetric prototype with radius $=1$ :

$$
\left[\begin{array}{lll}
W_{2} & W_{1} & W_{2} \\
W_{1} & W_{0} & W_{1} \\
W_{2} & W_{1} & W_{2}
\end{array}\right] g
$$

Specific prototypes can be seen in Fig. 6 .

Finally, a 3-layer example is given in Fig. 7 for receptive field interactions. Cells constituting the three layers have different time constants $\left(\tau_{1}, \tau_{2}, \ldots\right)$. Layers are interconnected via feedforward Gaussian templates $\left(T_{1}, T_{2}, \ldots\right)$ with various radii and "shapes" $\left(r_{1}, r_{2}, \ldots ; \sigma_{1}, \sigma_{2}, \ldots\right)$ forming the receptive fields (RF1, RF2 ...). Moreover, the

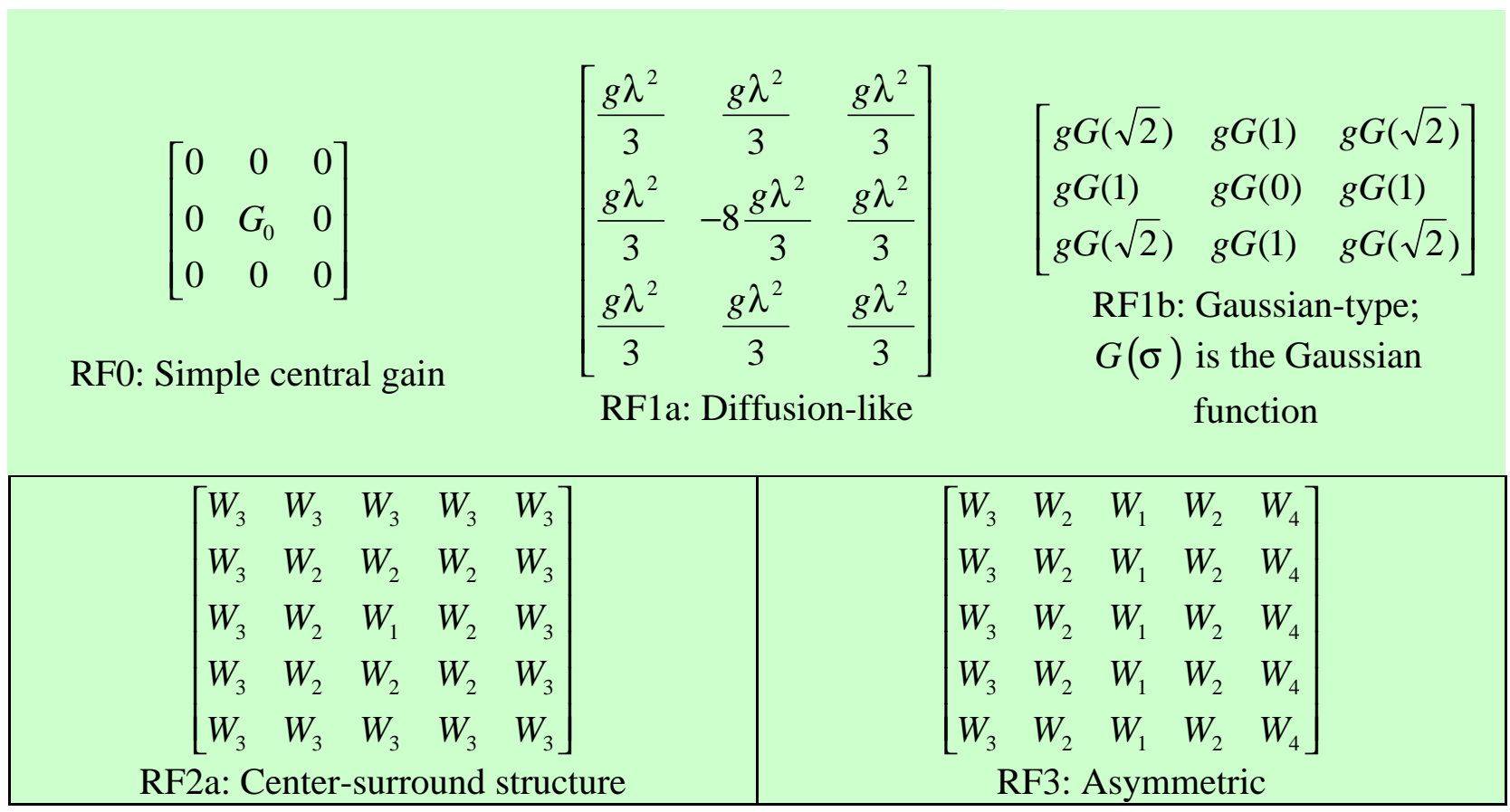

Fig. 6. Receptive field prototypes. The structure is defined by the pattern of weights in the representative template. $g$ is a general weight factor. RF1a belongs to the so-called reaction-diffusion template class (a detailed description can be found in [Chua, 1997]). 


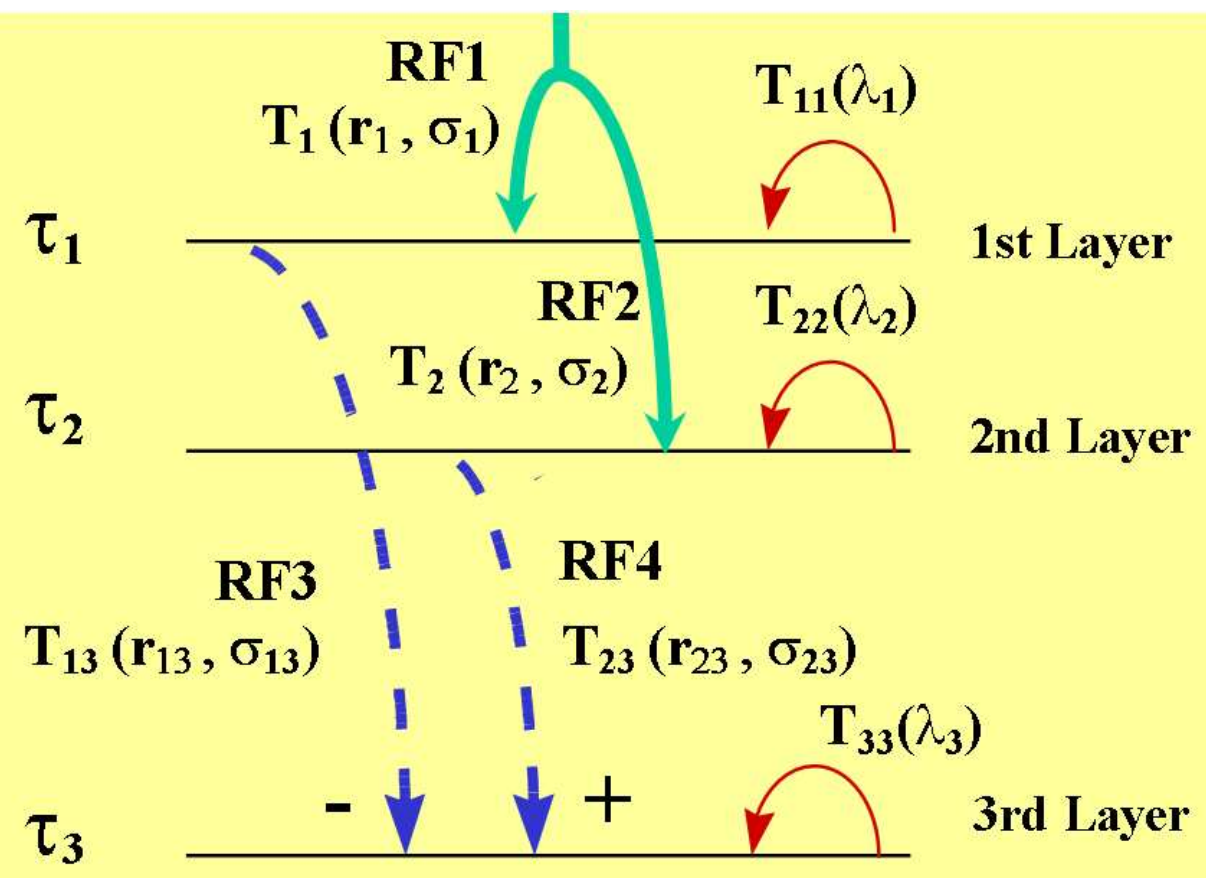

Fig. 7. A 3-layer receptive field interaction example $\tau_{1,2,3}$ : time constants; $T_{1,2,3,13,23}$ : feedforward templates; $r_{1,2,3}$ : size of templates; $\sigma_{1,2,3}$ : shape parameter of the templates; RF1,2,3,4: receptive fields; $T_{n n}$ : self-feedback diffusion templates.

self-feedback diffusion type templates $\left(T_{11}, T_{22}, \ldots\right)$ can tune the dynamics of this system as well.

\section{A Single "Naive" Retina Model as a Prototype Description}

\subsection{A single-layer CNN retina model}

The retina has five major classes of neurons: photoreceptors, horizontal cells, bipolar cells, amacrine cells and ganglion cells. These cells are arranged in three layers and they synapse in two synaptic layers. The photoreceptors, bipolar and horizontal cells contact each other in the outer plexiform layer (OPL) while the bipolar, amacrine and ganglion cells make synaptic connections in the inner plexiform layer (IPL) [Dowling, 1987; Werblin, 1991; Teeters \& Werblin, 1991]. Similarly to the bipolar cells, the retinal ganglion cells could respond to the light stimulus by "ON" (depolarization) or "OFF" (hyperpolarization) showing antagonistic "center-surround" receptive field characteristics. A schematic representation of the vertebrate retina is given below (see Figs. 8 and 9).

The model presented here is just to introduce a typical modeling technique. The modelers usu- ally choose a sensible complexity depending on the function(s) they expect the model to reproduce, and within this framework, the implementation is formed to be as close to the real biological situation as possible.

The following very simple retina model consists of a single layer instead of the ten classical neuroanatomical layers, and is able to reproduce some elementary spatial (detection of outlines and edges with arbitrary direction) and temporal effects.

The B template [see Eqs. (7)-(12)] simulates the spatial-type (spatial organization related, e.g. center-surround antagonism) analysis of visual input characterizing the OPL. The model exploits the fact that ganglion cells have an aggregated Difference-of-Gaussians (DOG) spatial receptive field [Linsenmeier et al., 1982; Roska et al., 1993b], where signals from the center and surrounding regions are separately summed, and the resulting center and surrounding signals have antagonistic effects on the ganglion output signal (RF-2a receptive field prototype).

The model also involves a very simple aggregate form of the IPL in the form of a delayed type A template [see Eq. (6)] providing a temporal-type 


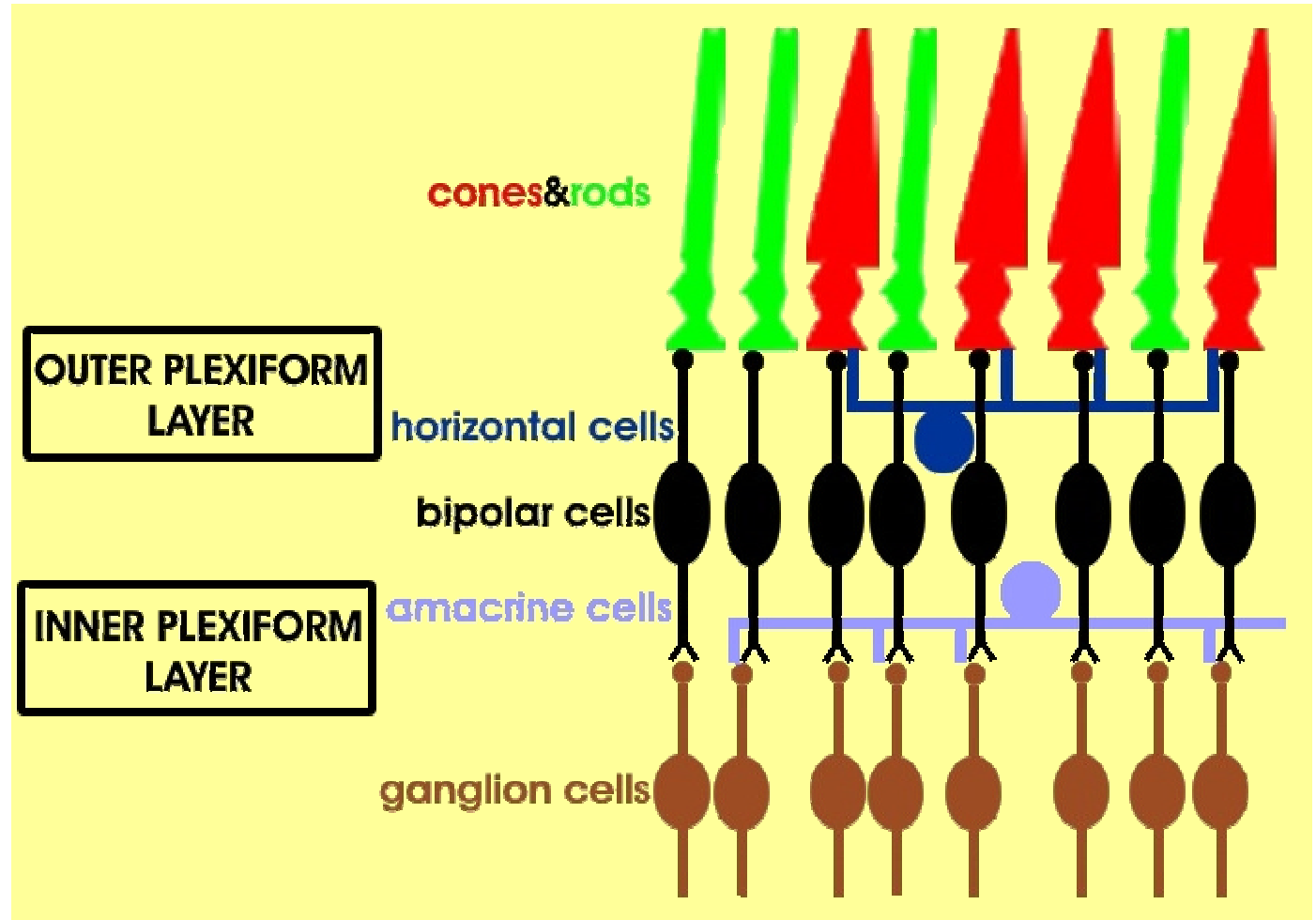

Fig. 8. Schematic organization of the vertebrate retina.

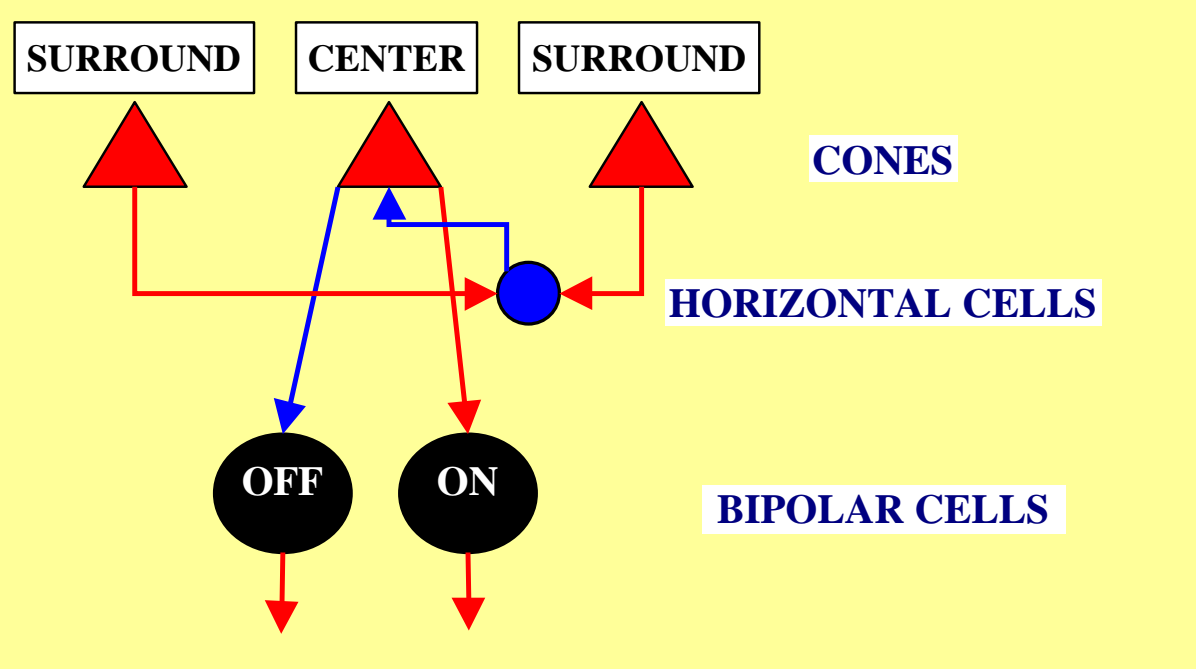

Fig. 9. Symbolic representation of the outer plexiform layer of the retina. Blue lines: inhibition. Red lines: excitation (see [Kandel et al., 2000b]). 
analysis.

$$
\begin{gathered}
A=[0] \\
A^{\tau}=\left[\begin{array}{rrrrrrr}
0.0 & 0.0 & 0.0 & 0.0 & 0.0 & 0.0 & 0.0 \\
0.0 & -0.1 & -0.1 & -0.1 & -0.1 & -0.1 & 0.0 \\
0.0 & -0.1 & -0.3 & -0.3 & -0.3 & -0.1 & 0.0 \\
0.0 & -0.1 & -0.3 & 3 & -0.3 & -0.1 & 0.0 \\
0.0 & -0.1 & -0.3 & -0.3 & -0.3 & -0.1 & 0.0 \\
0.0 & -0.1 & -0.1 & -0.1 & -0.1 & -0.1 & 0.0 \\
0.0 & 0.0 & 0.0 & 0.0 & 0.0 & 0.0 & 0.0
\end{array}\right] \\
B=\left[\begin{array}{lllllll}
G(3) & G(3) & G(3) & G(3) & G(3) & G(3) & G(3) \\
G(3) & G(2) & G(2) & G(2) & G(2) & G(2) & G(3) \\
G(3) & G(2) & G(1) & G(1) & G(1) & G(2) & G(3) \\
G(3) & G(2) & G(1) & G(0) & G(1) & G(2) & G(3) \\
G(3) & G(2) & G(1) & G(1) & G(1) & G(2) & G(3) \\
G(3) & G(2) & G(2) & G(2) & G(2) & G(2) & G(3) \\
G(3) & G(3) & G(3) & G(3) & G(3) & G(3) & G(3)
\end{array}\right] \quad B^{\tau}=[0]
\end{gathered}
$$

$$
\begin{gathered}
z=0 ; \quad \tau=3 ; \\
G(r)=G_{C}(r)-G_{S}(r) ; \\
G_{c}(r)=k_{c} e^{-\left(r / p_{c}\right)^{2}} ; \quad G_{s}(r)=k_{s} e^{-\left(r / p_{s}\right)^{2}} \\
k_{C} / k_{S}=\left(r_{C} / r_{S}\right)^{2}=9, \quad p_{C}=0.7, \\
p_{S}=2.1, \quad k_{C}=5, \quad k_{Z}=0.55 \\
G(0)=4.45, \quad G(1)=0.2, \\
G(2)=-0.222, \quad G(3)=-0.07 .
\end{gathered}
$$

The discovery of the details of the inner plexiform layer shed new light in retinal functions [Roska \& Werblin, 2001] as well as their modeling [Bálya et al., 2002]. Their details and their CMOS chip implementation are described in the companion paper of this issue [Werblin \& Roska, 2004; Bálya et al., 2004].

\section{The Visual Thalamus (LGN)}

The axons of the retinal ganglion cells travel together in the optic tract on the basal surface of the frontal lobe, and innervate the visual thalamic nucleus (lateral geniculate nucleus, LGN). Axons of the LGN project to the primary - and partly the secondary - visual cortical areas, maintaining the precise topographic map of the sensory periphery (the retina).
However, the thalamus is not merely a simple relay station, but an intelligent switch with dynamic signal processing capabilities, under the control of cortical feedback and other (secondary visual pathway) mechanisms [Marr, 1982].

Most relay neurons have similar receptive fields like the so-called retinal X or Y ganglion cells which innervate them, and are also called $\mathrm{X}$ or $\mathrm{Y}$ type cells. While $\mathrm{X}$ cells have smaller dendritic arbor and receptive field, these cells respond better to visual stimuli of higher spatial frequency, Y type relay cells bear with a relatively wide receptive field due to their expansive dendritic tree. In addition to the relay neurons, there are inhibitory cells in and beside the thalamic visual nucleus: local interneurons and neurons in the perigeniculate (reticular thalamic) nucleus [Kandel, 2000c].

The innervation of the visual thalamic cells is somewhat surprising, only about $20-25 \%$ of the axons comes from the retina, while a massive projection - more than $50 \%$ in the cat - arrives from the primary and secondary visual cortical areas.

The authors present a simplified connectivity model of the X type cells (see Fig. 10) [Kandel et al., 2000c; Roska et al., 1993b], which frequently give collaterals to the inhibitory neurons (described above), mainly to the perigeniculate nucleus, which in turn, has a strong projection to the LGN.

Figure 10 shows the synaptic connections of a simplified LGN model and the corresponding incomplete, "decorticated" 5-layer CNN architecture 


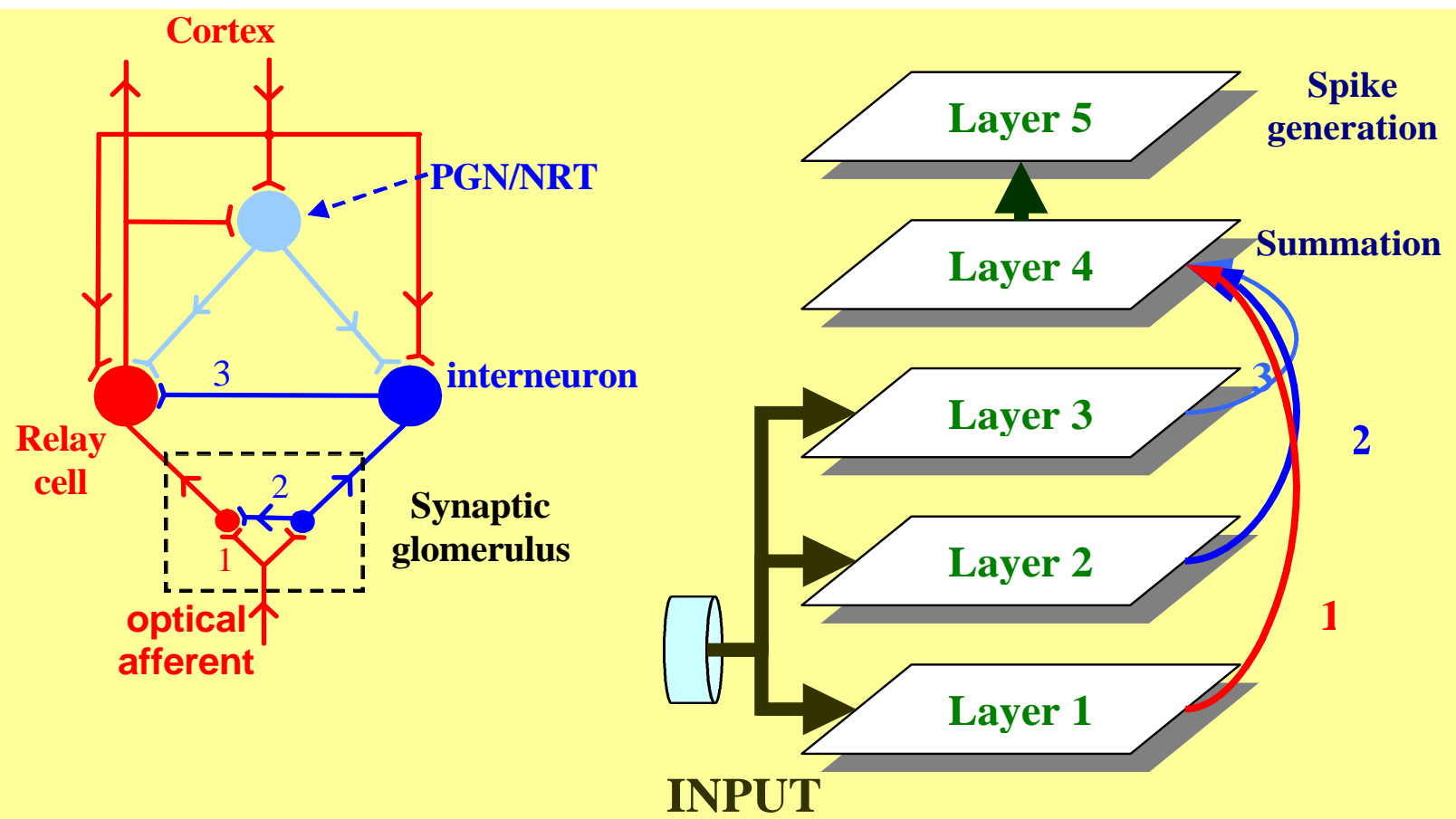

Fig. 10. (a) Synaptic connections of a simple LGN model. (b) A five-layer model and the corresponding connections of a relay cell is presented. The first three layers (and their feedforward connections to layer 4) implements complex input channels of the cell: the first (red line) serves as excitatory, the second and the third (blue lines) form fast and slow inhibitory inputs, respectively. Innervations coming from the cortex and the perigeniculate nucleus are not represented here.

of a relay cell. The synaptic glomerulus, which consists of a special synaptic arrangement (the so-called "triadic synapse") [Hámori et al., 1974], forms the center of the design. The applied synapse models use almost all the available synapse prototypes like S1a, S1b, S2a and S4. Moreover, the model uses some more complicated nonlinear interactions enabling the structure to generate "spikes" (action potentials, see Sec. 2). The receptive field prototypes used can be classified as RF-0 and RF-3.

A single "biological" cell is represented by several CNN cells and their connections throughout the five layers. The first three layers serve only as distinct input interfaces of the modeled LGN cell. They implement complex synaptic mechanisms (e.g. different delays): an excitatory, and two inhibitory innervations with different dynamics. Layer 4 integrates the input signals and layer 5 generates spikes at various frequencies as a function of the incoming excitation from layer 4 .

CNN templates of the simplified LGN model can be seen in Figs. 11-15. The 5-level structure with the special delay type nonlinearities enable two distinct operating modes [Reinagel et al., 1999].
In the so-called "tonic mode" incoming signals are transmitted faithfully, while in "burst mode" the cells generate periodic patterns of bursts in response to certain stimulation patterns.

\subsection{Center-surround receptive field properties of LGN cells}

Figure 16 demonstrates the center-surround property of some LGN cells. The picture shows two different stimuli (top) and the response transients of an On-center Off-surround LGN cell using the above studied 5-layer CNN model. In the first case (left) the whole center is dark and the surround is illuminated, while in the second case (right) it is the opposite. The second stimulus elicits a vigorous response in the On-center cell, while the first stimulus evokes practically no response.

\section{Simple Cortical Cells and Layers}

Cortical nerve cells are organized into $0.5-1.0 \mathrm{~mm}$ wide regions, referred to as columns. These are both physiological and anatomical units: neurons within a cortical column have similar receptive field 


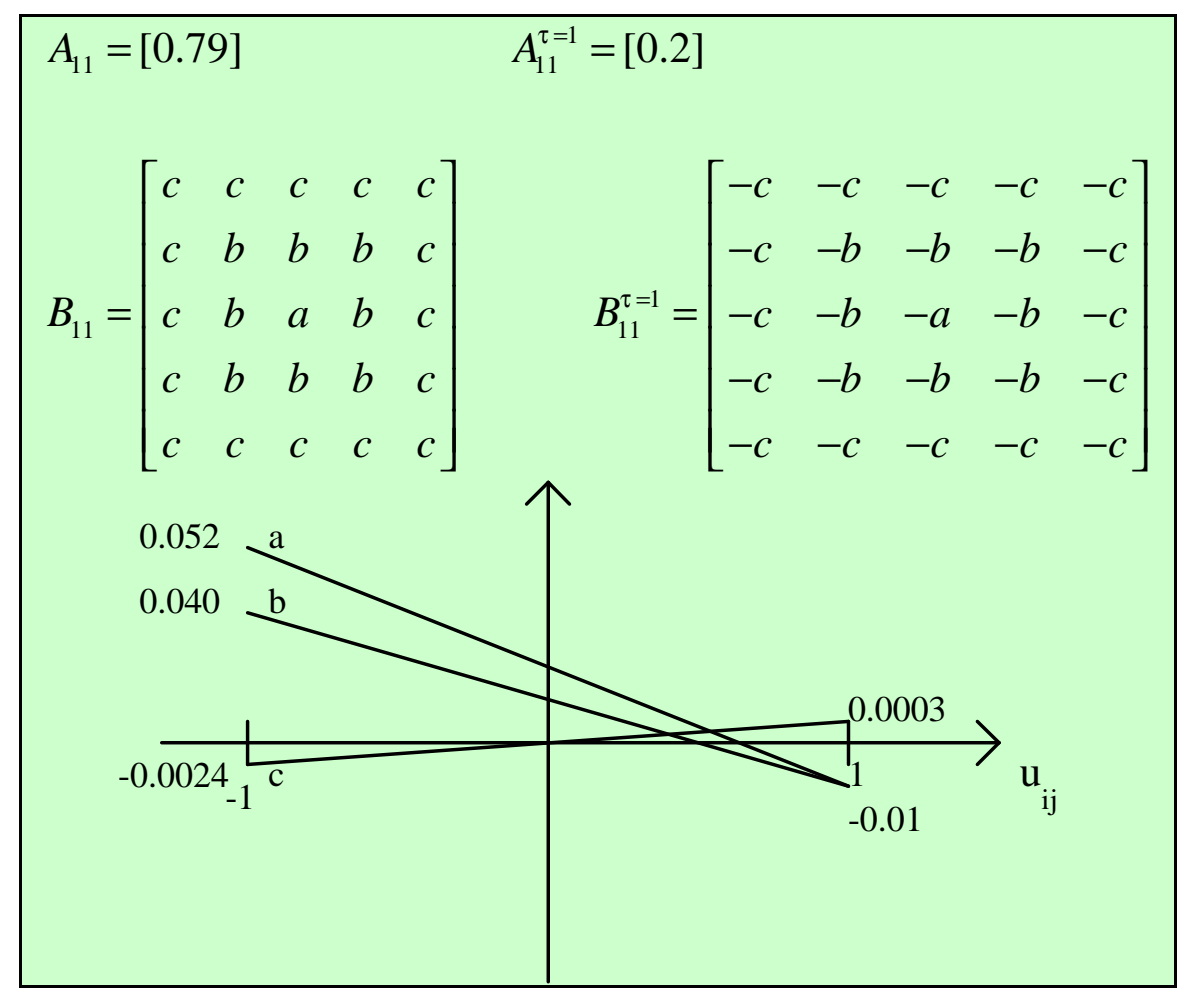

Fig. 11. Layer 1 (see Fig. 10) dynamics provides the central excitation component via template $B_{11}$. Transmitted signals decay quickly due to the small $\tau$ of the delay type $B_{11}^{\tau=1}$ template.

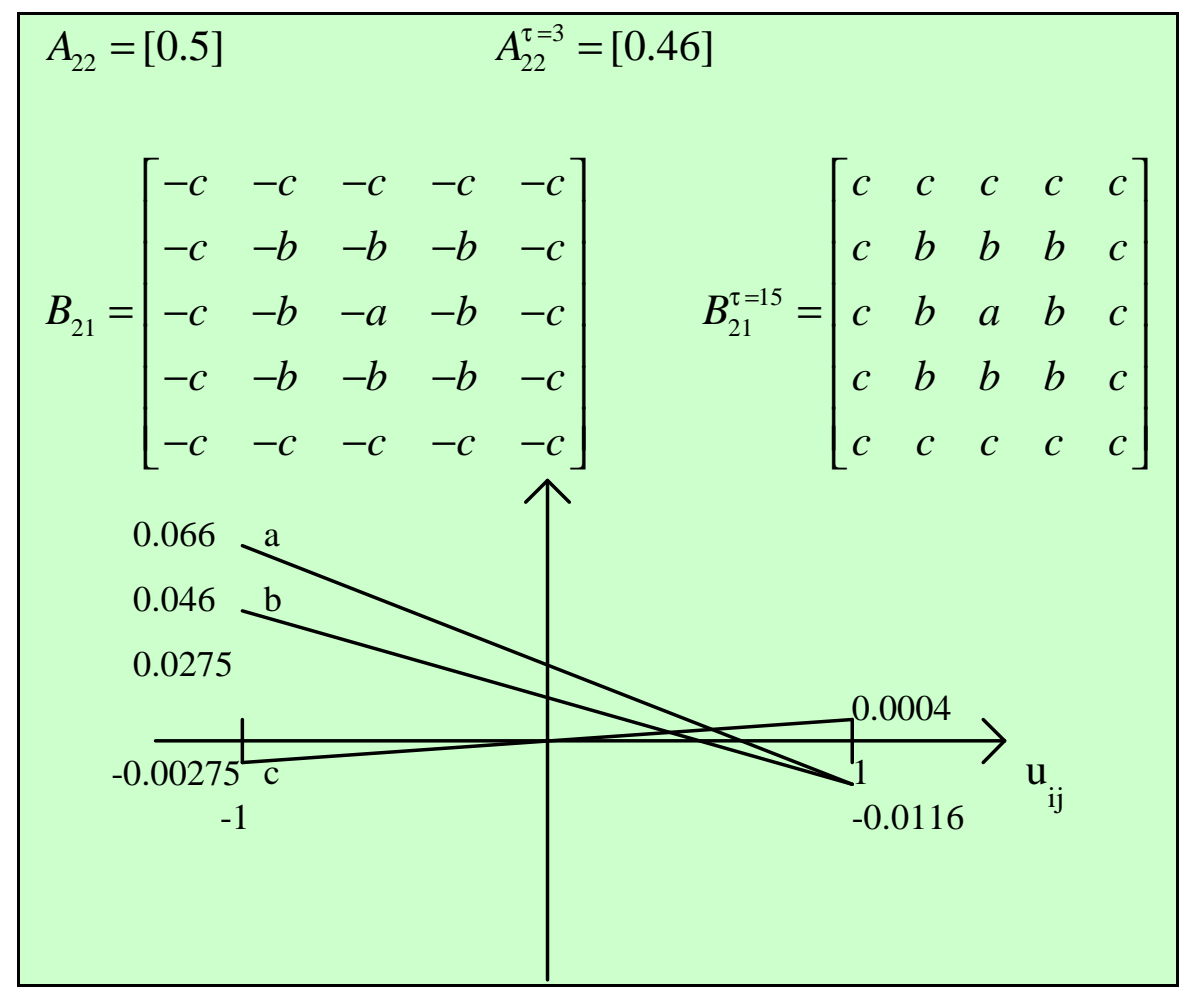

Fig. 12. Layer 2 (see Fig. 10) serves as a fast inhibitory channel. Its effect dies out faster than the inhibition of layer 3 but later than the excitation from layer 1 . 


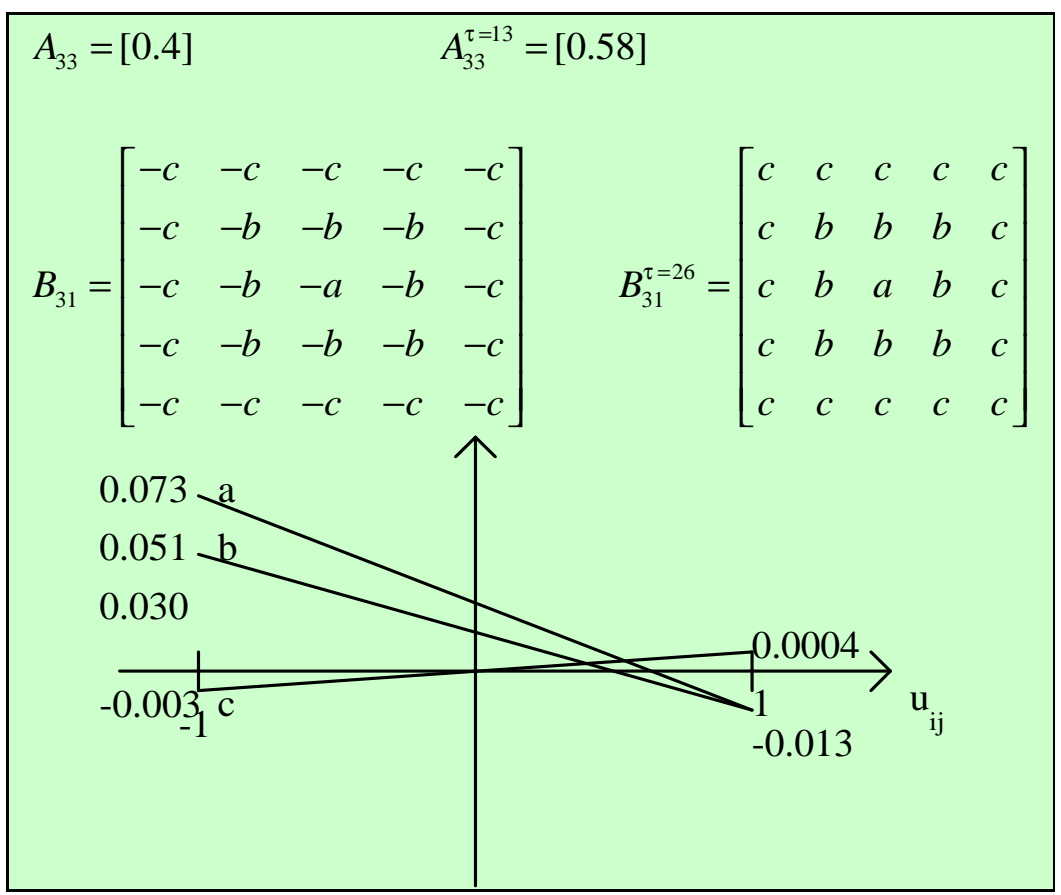

Fig. 13. Layer 3 (see Fig. 10) produces prolonged inhibition.

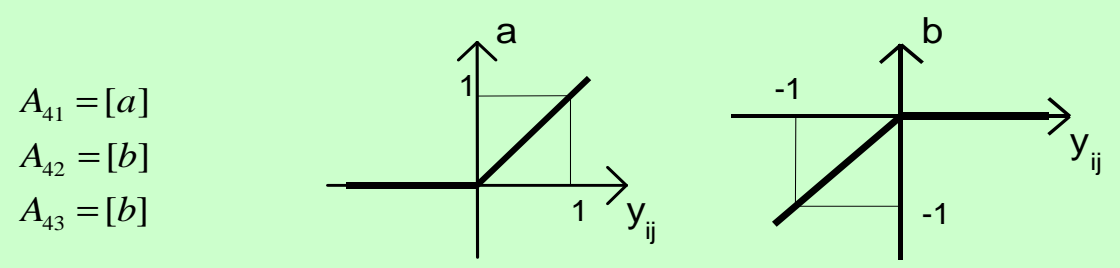

Fig. 14. Layer 4 (see Fig. 10) integrates the signals coming from the excitatory (21) and inhibitory $(2,3)$ layers.

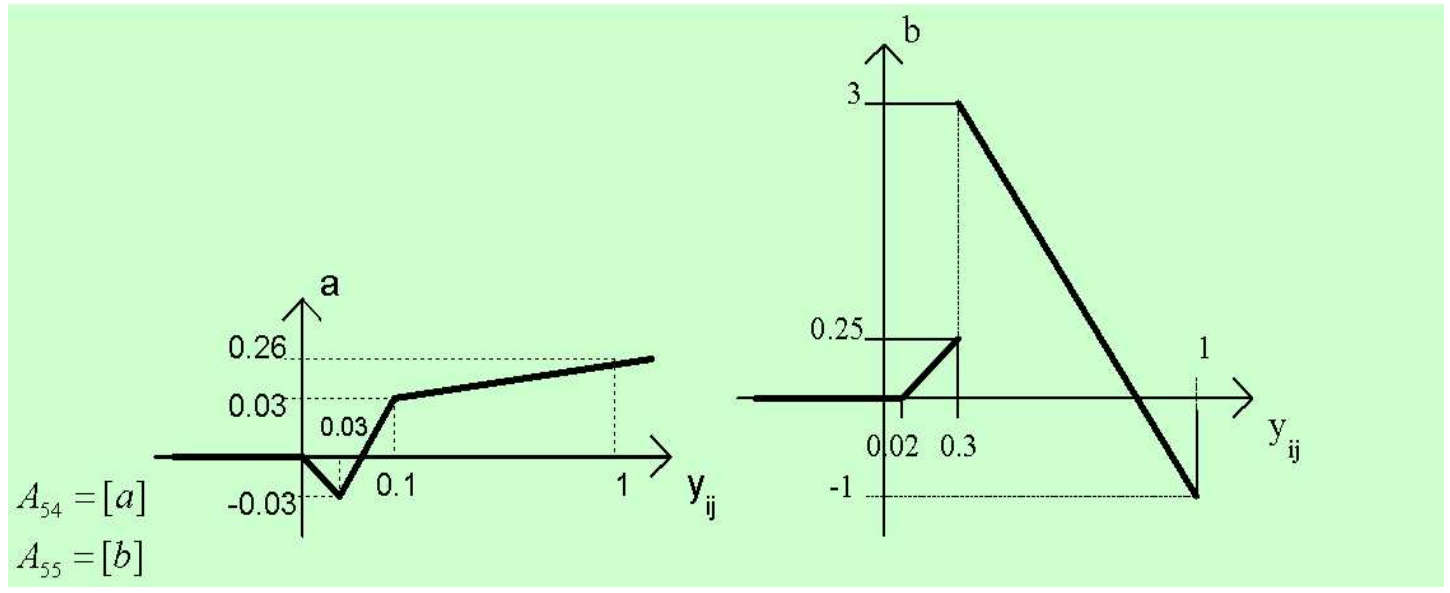

Fig. 15. Layer 5 helps in generating spikes as a function of the output level of layer 4 . 

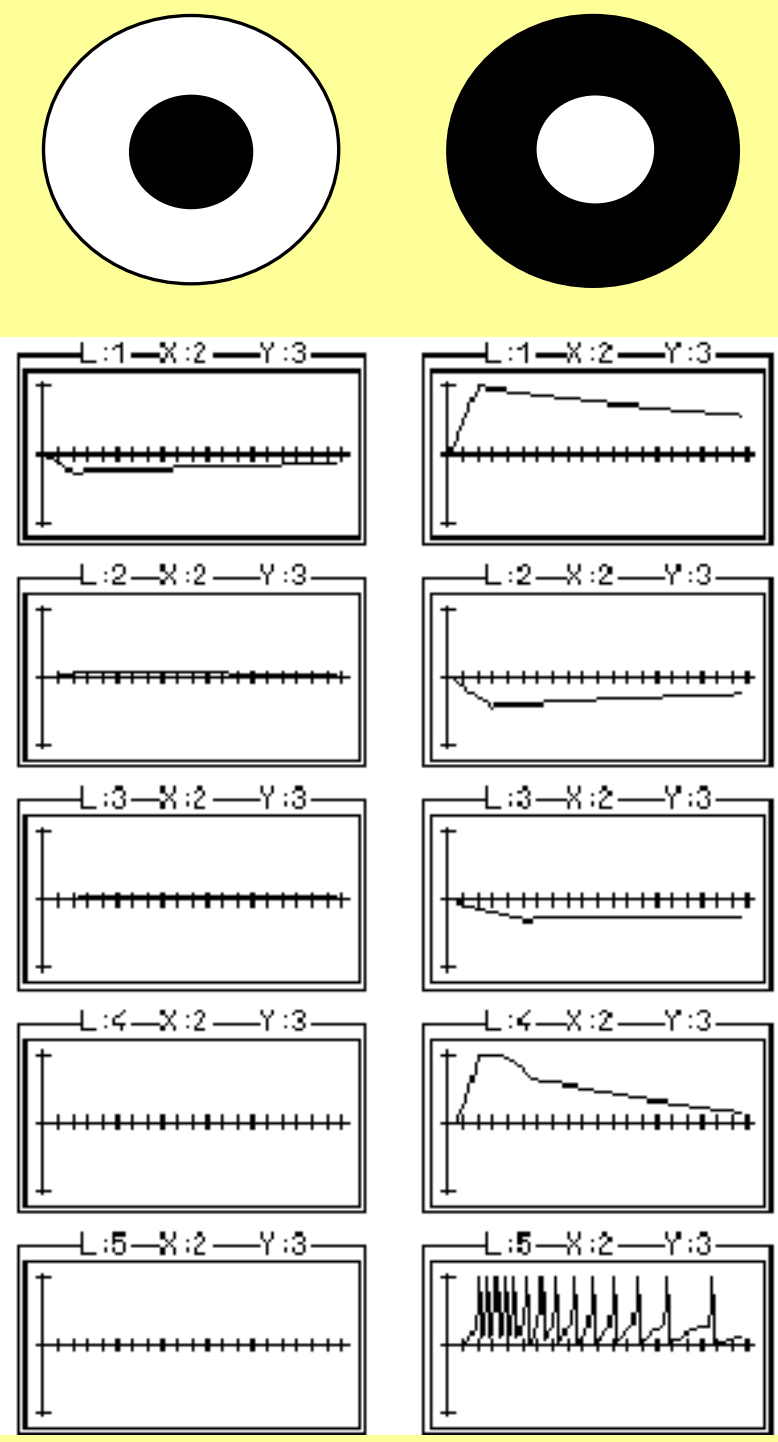

Fig. 16. Center-surround property of an artificial on-center off-surround LGN cell. The graphs show the activities (vertical axis) of the different layers (L1-5) in a cell versus time (horizontal axis). In the left column the whole center is dark and the surround is illuminated, in the second case (right) it is vice versa. The second stimulus elicits a vigorous response.

characteristics. Visual information is transmitted to these columns along parallel, labeled paths. Parallel paths maintain a kind of division of labor. A number of maps generated from retinal ganglion cells can be found in the visual system of higher mammals. Visual entities like color, form and motion are transmitted via separate channels and processed in separate columns [Kandel et al., 2000c].

At the same time, retinotopic maps are well preserved at each level of the retino-geniculocortical paths. The morphological basis of these maps is the strictly positioned topographic pattern of neurons at each level. Lateral mechanisms of processing are also found at all levels, maintaining an integrated activity of smaller or larger groups of neurons. The most common mechanism of this is the center-surround activation-inhibition, to enhance spatial or chromatic contrast.

Towards higher-level areas along the visual neuraxis, the cells respond to more and more complex stimuli and their receptive fields grow significantly.

In the cat primary visual cortex Hubel and Wiesel [1962] described two broad classes of neurons, simple cells and complex cells. Response properties of the simple cells show close relationship to the receptive field structure of retinal ganglion cells, 


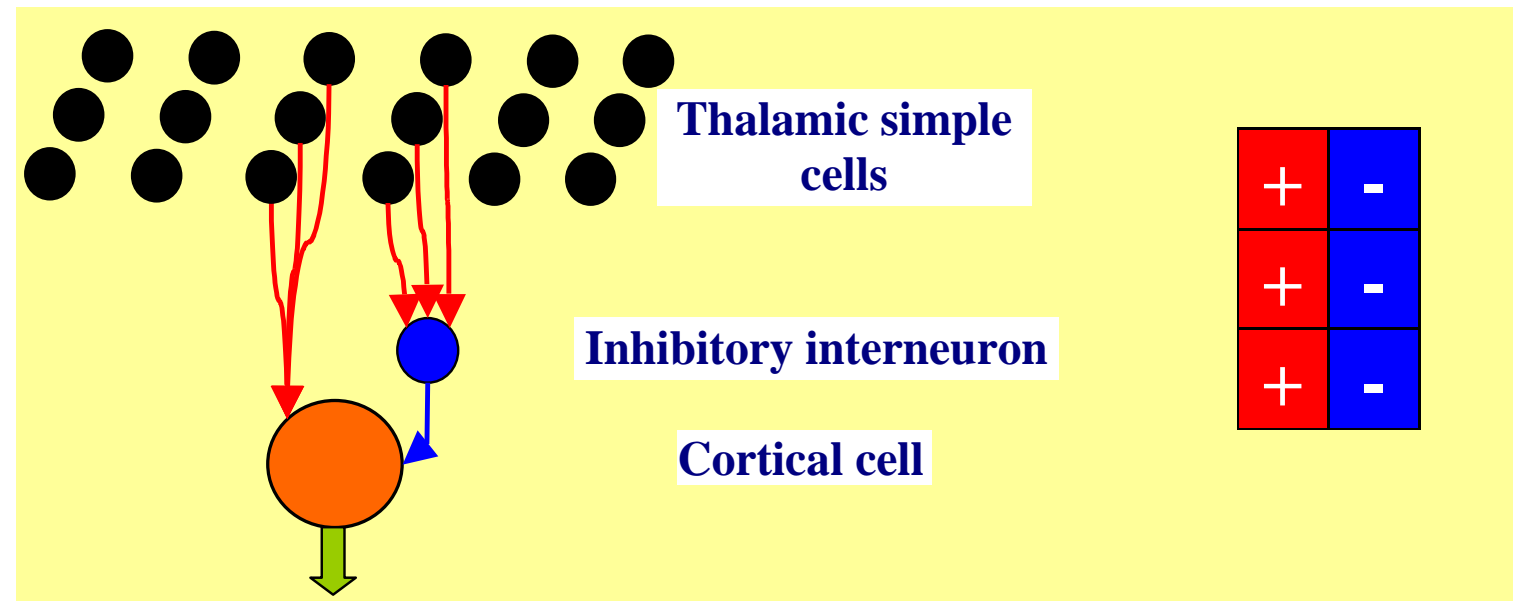

Fig. 17. Schematic diagram of two converging thalamocortical projections, and the resulting receptive field of the cortical cell (on the right side).

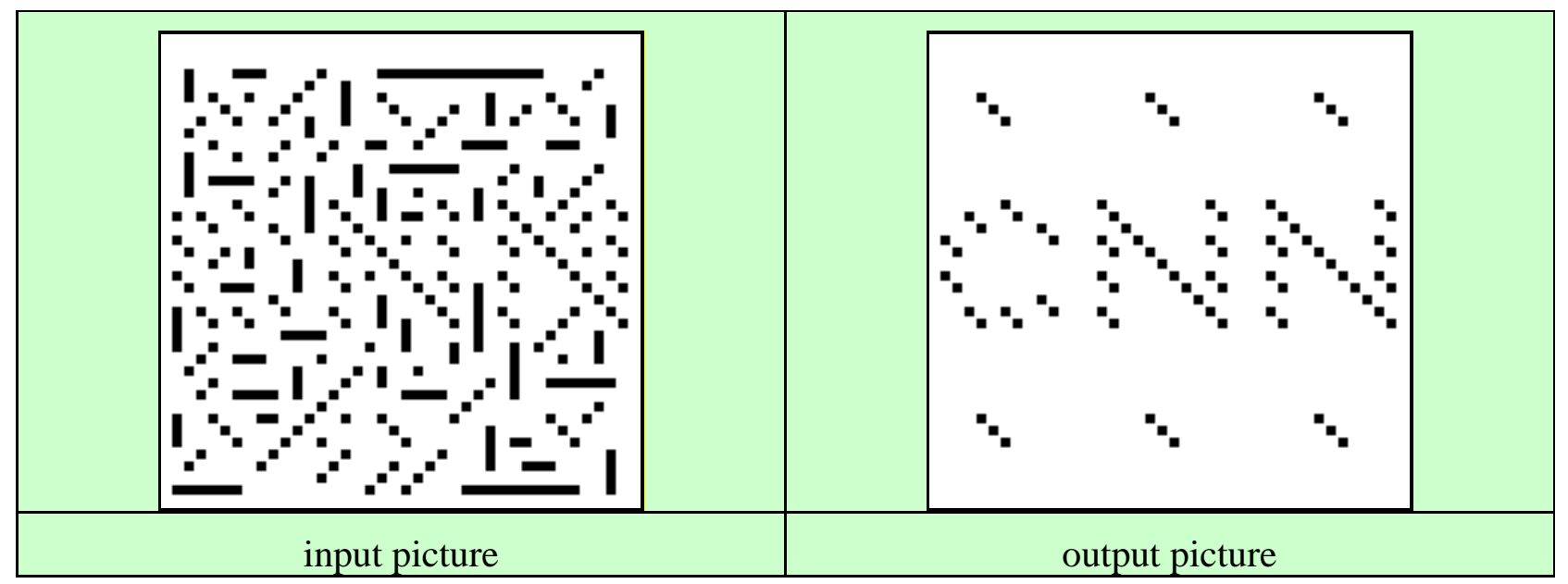

Fig. 18. Processing of an image with orientation $\left(-45^{\circ}\right.$ slope $)$ selective CNN cells.

or to thalamic relay cells. One difference is that the spatial structure of these cells is not rotational invariant. That is, they prefer special stimulus orientations, and have separated "on" and "off" subregions. Some of them are direction sensitive as well: their activity is highly influenced by the motion parameters of stimuli crossing their RFs. Complex cells also respond to stimuli at one orientation, but their receptive field is not segregated into on and off subregions. Simple cells can be found more frequently in layers that receive direct thalamic input.

An aspect of the simple cells' behavior, whose receptive fields are asymmetric and do not follow the center-surround antagonistic scheme, can be modeled by an appropriate convergence (RFI-2 pro- totype) of simple thalamic cells shown in Fig. 17. The receptive fields of the $\mathrm{X}$ type cells are organized in two neighboring lines.

\subsection{Orientation sensitivity}

Below a CNN template is given which selects lines with a $-45^{\circ}$ slope, and the simulation is displayed with the input and output pictures in Fig. 18.

CNN template:

$$
A=[2] \quad B=\left[\begin{array}{ccc}
0.25 & 0 & 0 \\
0 & 0 & 0 \\
0 & 0 & 0.25
\end{array}\right] \quad z=-1
$$




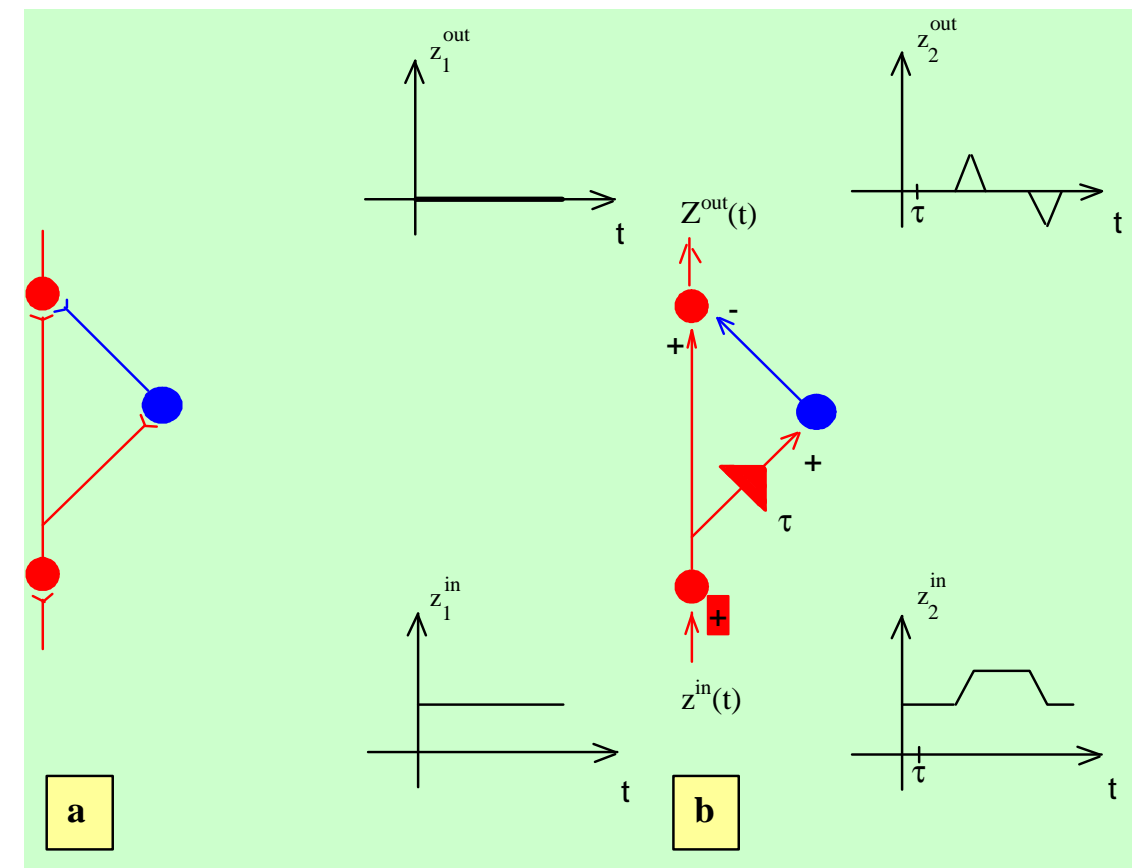

Fig. 19. (a) Neural motion detection architecture: triadic synapse arrangement. (b) Processing of stationary and dynamic signals by the model of the triadic synapse. $z_{\text {in }}(t)$ denotes the input signal from the sensory periphery, $z_{\text {out }}(t)$ is the output level of the detector system.

\subsection{Direction sensitivity}

One of the neural motion detection architectures is the triadic synaptic arrangement [Lábos et al., 1990], given in Fig. 19(a). Red circles depict excitatory cells, the blue ones represent inhibitory interneurons. In Fig. 19(b) an artificial model of the triadic synapse is shown with two input signals (bottom) and the corresponding responses (top). The red triangle indicates the delay of the inhibitory interneuron.

CNN templates corresponding to the triadic synapse (RF-0 receptive field and S1b synapse prototypes):

$$
\begin{gathered}
A=[0] \quad B=[a] \quad A^{\tau}=[0] \quad B^{\tau}=[-b] \\
z=0 \quad \tau=1 \quad a, b>0 ; \quad \text { example: } a=b=1
\end{gathered}
$$

\subsection{Direction selective neural connection scheme}

Several neurons in the cortex show certain direction selective properties. This results from some special spatial neural connections. Figure 20(a) gives a concept diagram used in the study of Sillito and Murphy [1988] illustrating a neural connection scheme which might generate direction selectivity of moving stimuli. There are feedforward inhibitory connections directed to the right which cause a wave of inhibition suppressing responses to stimuli moving to the right. In addition, the feedback excitatory connections to the opposite direction reinforce the stimulus response in this direction. Thus, the upper neurons select the stimulus moving to the left. Figure 20(b) shows an artificial representation of the direction selective connection scheme together with two stimuli moving in opposite directions (bottom) and the responses (top).

The CNN template corresponding to the direction selective neural connection scheme (RF-3 prototype):

$$
\begin{gathered}
A=[0] \quad B=\left[\begin{array}{lll}
0 & 0 & 0 \\
0 & a & b \\
0 & 0 & 0
\end{array}\right] \\
A^{\tau}=[0] \quad B^{\tau}=\left[\begin{array}{ccc}
0 & 0 & 0 \\
-c & 0 & 0 \\
0 & 0 & 0
\end{array}\right] \\
z=0 \quad \tau=1
\end{gathered}
$$

$a, b, c>0 ; \quad$ example: $a=c=1.5 ; \quad b=1$

Example of a direction selective motion detection where only points (of the input) which move in a given (in this case: right) direction are detected: 


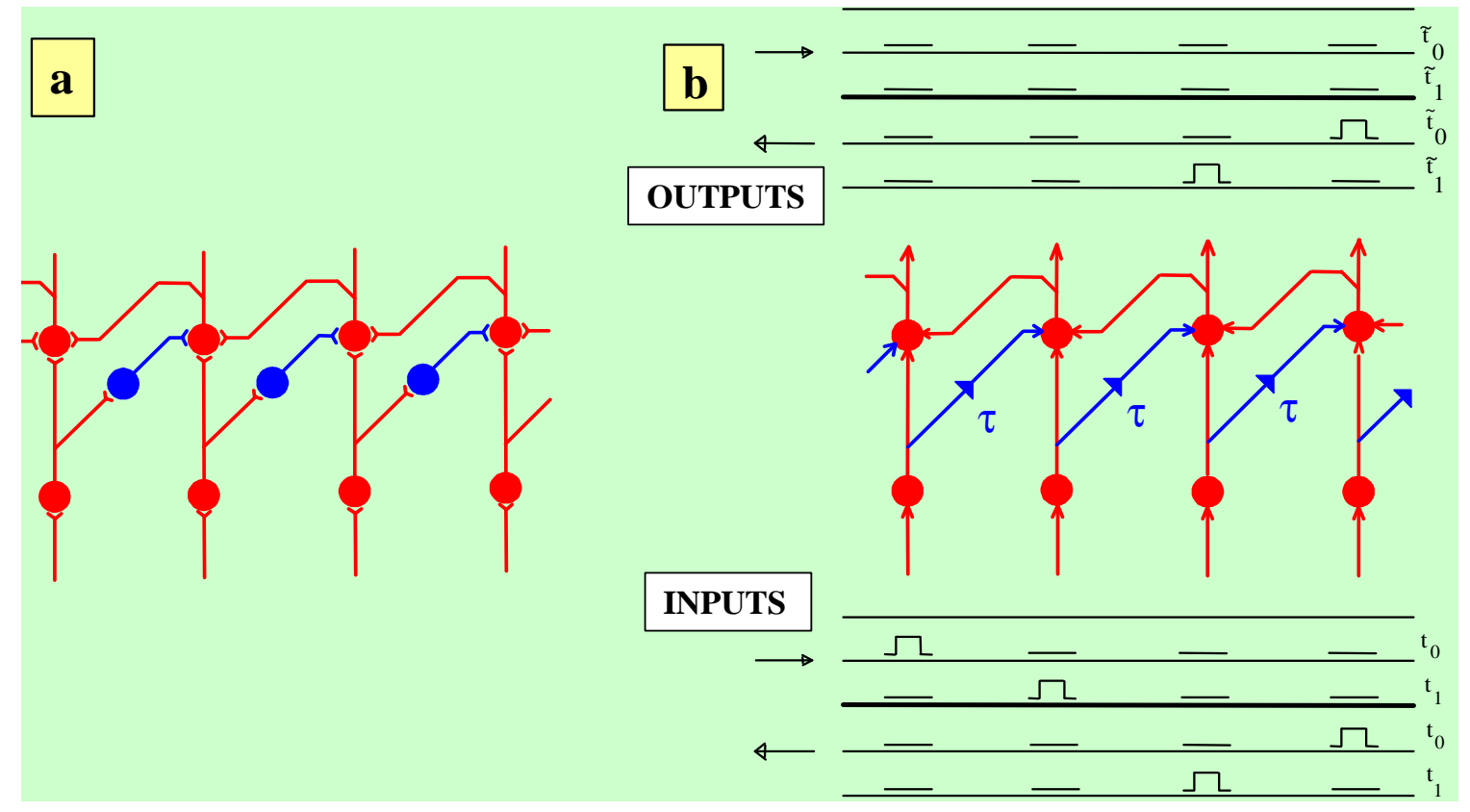

Fig. 20. Neural connection scheme having direction selectivity for moving stimuli.

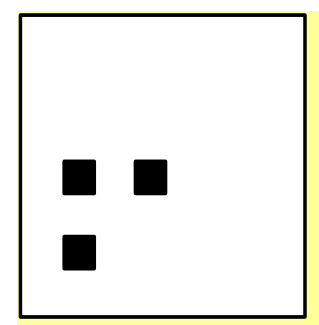

input 1

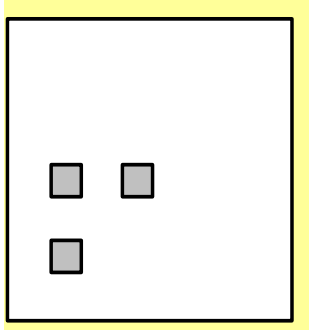

output 1

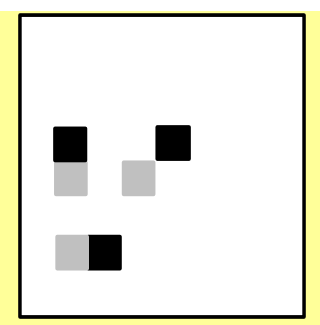

input 2

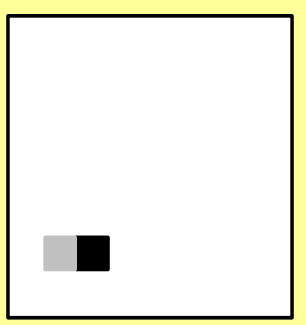

output 2

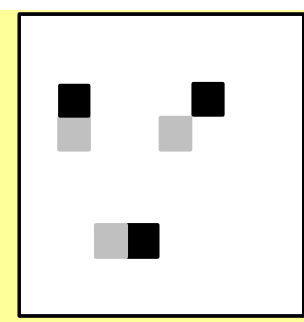

input 3

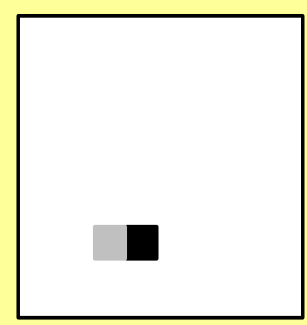

output 3

Fig. 21. Direction selective motion detection. Three consecutive snapshots of the movement. 


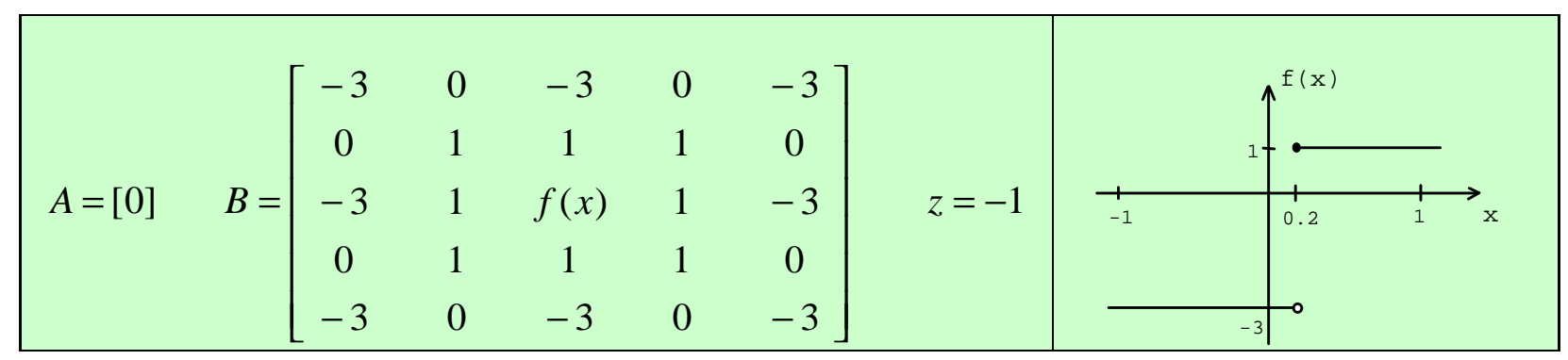

Fig. 22. CNN template for length tuning.

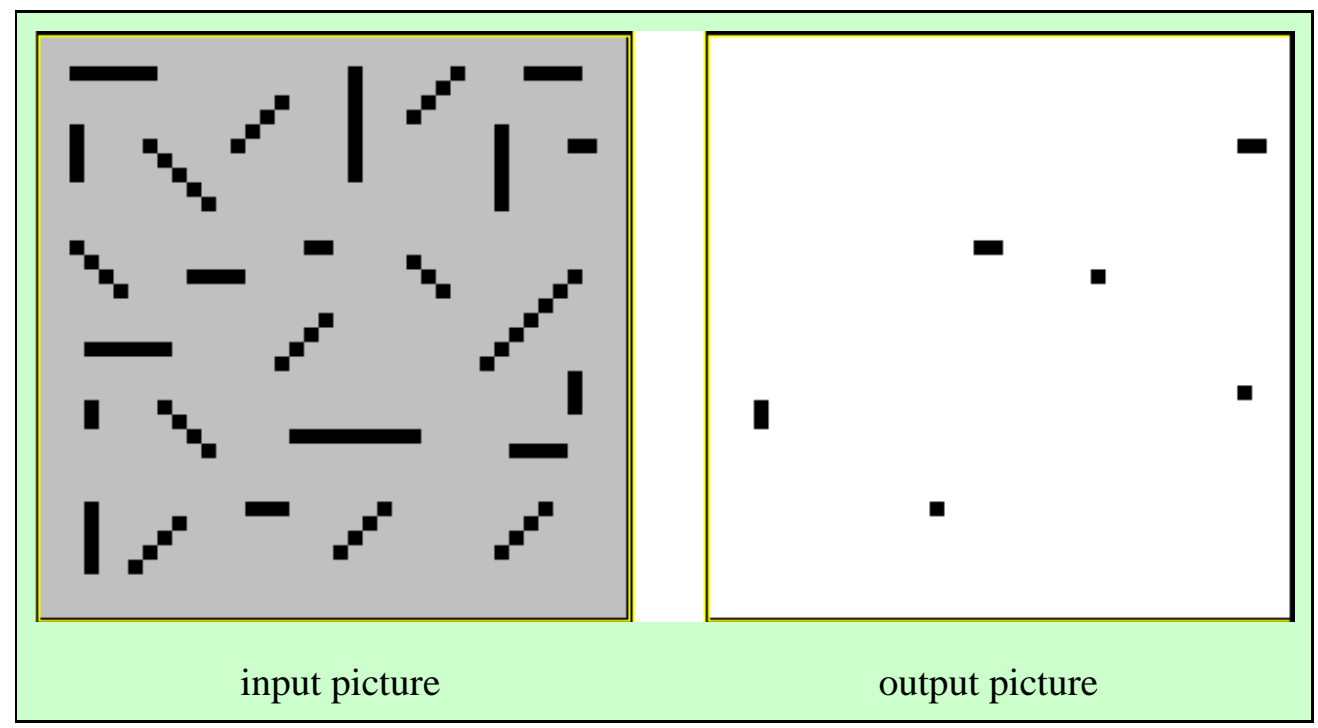

Fig. 23. Length tuning: processing of bars with different lengths.

the input and output images were both sampled, three snapshots from both the input and output are displayed in Fig. 21.

The CNN template used here:

$$
\begin{gathered}
A=[0] \quad B=\left[\begin{array}{ccc}
0 & 0 & 0 \\
0 & 1.5 & 0 \\
0 & 0 & 0
\end{array}\right] \\
A^{\tau}=[0] \quad B^{\tau}=\left[\begin{array}{ccc}
0 & 0 & 0 \\
1.5 & 0 & 0 \\
0 & 0 & 0
\end{array}\right] \\
-2.8<z<-1.6 \quad \tau=1
\end{gathered}
$$

\subsection{Length tuning}

Although certain neurons in the visual cortex give maximal response to an optimally oriented bar of a certain length, by increasing this length their responses decrease or completely disappear [DeAngelis et al., 1994]. A possible explanation for this phenomenon might be that these "length sensitive" neurons receive an excitatory input from the central region of their receptive field and an inhibitory input from cells spatially displaced to either side of the cell providing the main excitatory input.

In Fig. 23 a simple example of "length tuning" is given, where the input picture contains a number of bars of different lengths and the CNN template in Fig. 22 marks those whose length is less or equal to three pixels.

\section{Phenomenological Inferotemporal Model (Black Box)}

The anterior inferotemporal area of the monkey cortex - a later stage in the visual stream crucial for 


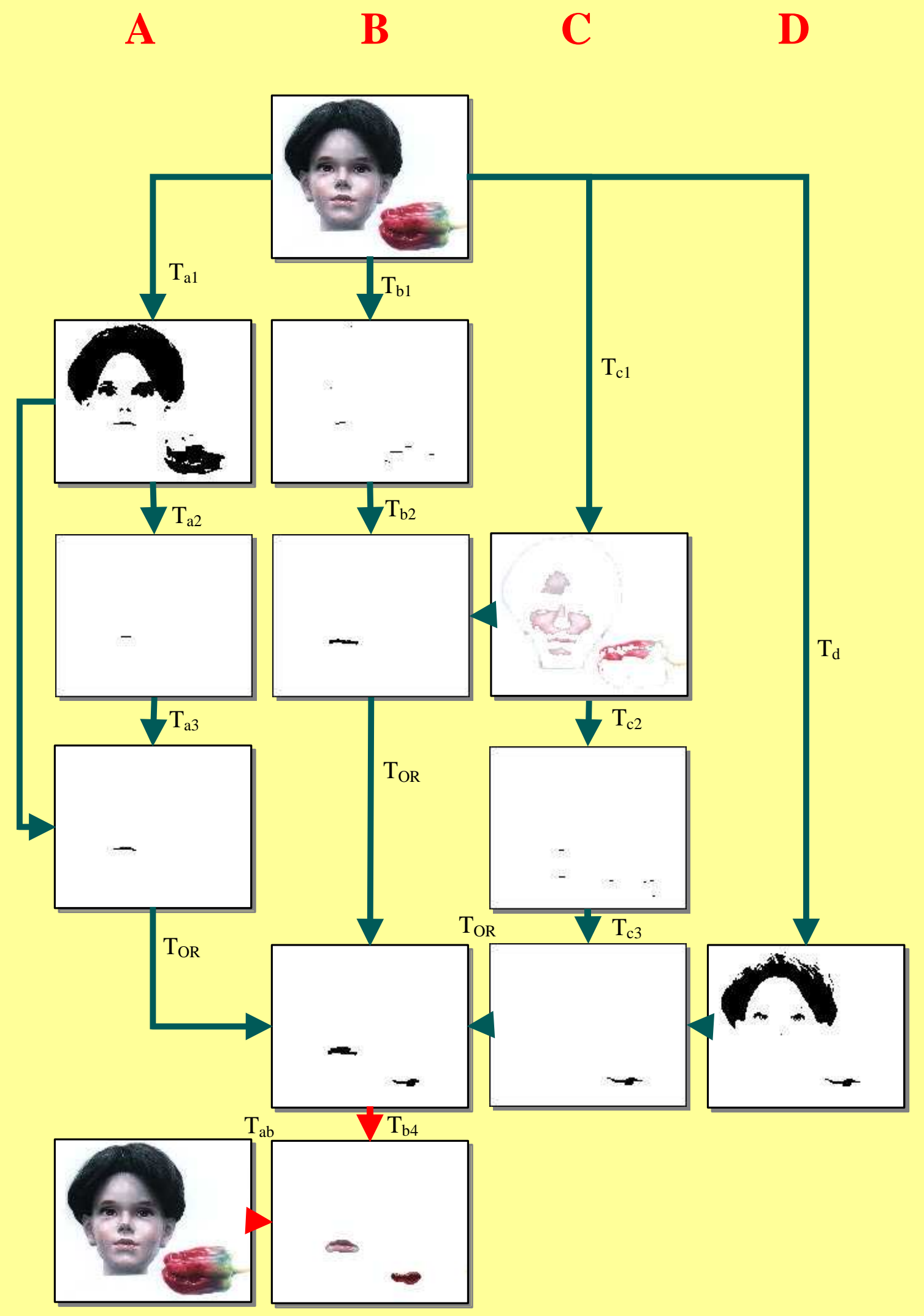

Fig. 24. Mouth detection in color pictures - on face and on pepper. $T$ denotes different template operations. Their functions are explained in the text. 
object recognition - has columnar arrangement, where each column is composed of groups of cells responsive to similar visual features of objects [Fujita et al., 1992]. However, it was also shown that optimal stimulus and tuning properties differed even between adjacent neurons of the same column. This finding suggests that the activation of a cortical column may encode a specific extracted feature, common to similar shapes (like a red mouth and a part of a red pepper), while the activity of individual neurons within the same column may assist in signaling even small variations of the same attribute. We show a phenomenological model implemented as analogic CNN algorithms: the method does not reflect the real situation in the brain, however, it gives a hint about the possible hierarchy of receptive field organizations and processing along the visual neuraxis.

\subsection{Mouth detection in color pictures - on face and on pepper}

Fujita and his colleagues [1992] reported some neurons which respond most strongly to the lips of a face. The study revealed that the stimulus feature critical for activation was a horizontally elongated shape with an upper half-dark, lower half-light pattern with the center. When a red pepper containing a mouth-like pattern was presented to the monkeys, the response of the "lips-selective" neurons was very strong again. A CNN analogic algorithm can reproduce this phenomenon, i.e. detects the mouth on a face and on the pepper as well [Lotz et al., 1995].
The steps of the algorithm are as follows ( $\mathrm{T}$ stands for different template operations.):

Detection of the upper lip (Fig. 24, column A).

Following the extraction of relatively dark patches in the original image by a threshold template (Eq. (17), $\left.T_{a 1}\right)$ a special template (Eq. (18), $T_{a 2}$ ) marks the upper lip. The structure of this template is similar to the shape of the lips of a mouth (horizontally elongated) and it marks longer and thin horizontal lines. Starting from the marking points the RECALL template $\left(T_{a 3}\right)$ restores the patch representing the upper lip.

$$
A=[2] \quad B=[0] \quad z=0.3
$$

$A=[0]$

$B=\left[\begin{array}{ccccccc}0 & 0 & 0 & 0 & 0 & 0 & 0 \\ 0 & 0 & 0 & 0 & 0 & 0 & 0 \\ -0.1 & -0.1 & -0.1 & -0.1 & -0.1 & -0.1 & -0.1 \\ 0.1 & 0.1 & 0.1 & 0.1 & 0.1 & 0.1 & 0.1 \\ 0.1 & 0.1 & 0.1 & 0.1 & 0.1 & 0.1 & 0.1 \\ -0.1 & -0.1 & -0.1 & -0.1 & -0.1 & -0.1 & -0.1 \\ 0 & 0 & 0 & 0 & 0 & 0 & 0\end{array}\right]$

$z=-2.4$

Detection of the lower lip (Fig. 24, column B)

First, an operation detects the "mouth" points in the black and white filtered original image using a template (see Fig. 25, $T_{b 1}$ ) whose structure is the same as the pattern to which the real cortical neurons respond strongly (lips of a face).

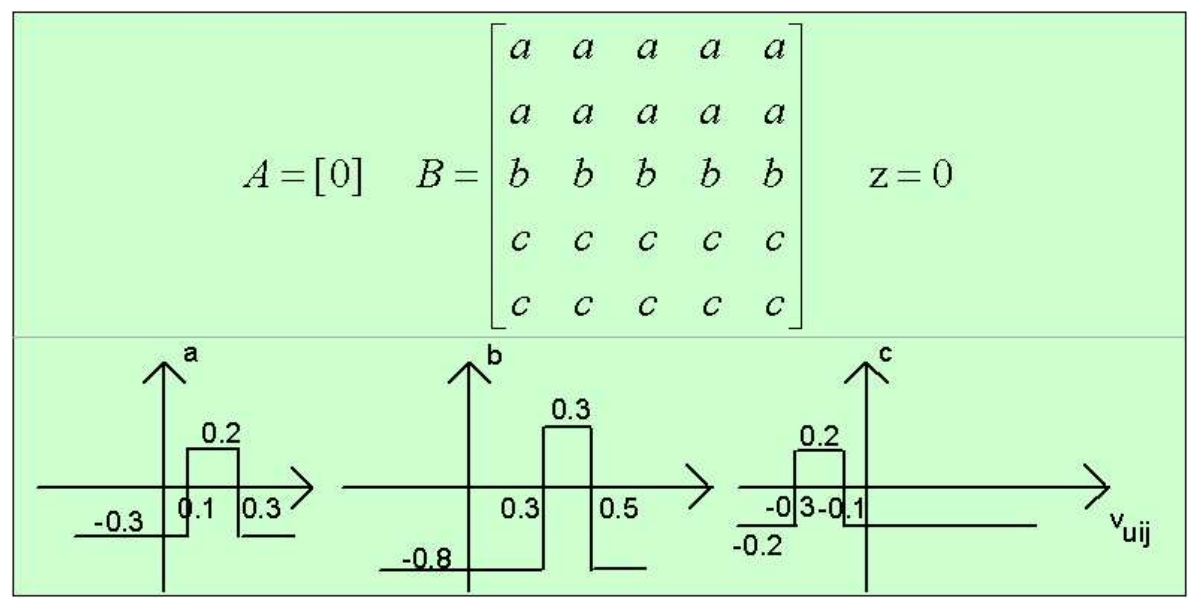

Fig. 25. Mouth detector template. 
The labeled pixels select and the RECALL template $\left(T_{b 2}\right)$ reconstructs the lower lip of the mouth in a red filtered $\left(T_{c 1}\right)$ and thresholded version of the original picture (Fig. 24, column C).

Recalling the mouth like pattern on the pepper (Fig. 24, column C, D)

A slightly modified version of the $T_{b 1}$ template $\left(T_{c 2}\right)$ selects the mouth-like pattern on the red filtered image $\left(T_{c 1}\right) . T_{c 3}$ recalls the whole mouth-like pattern from a thresholded transformation $\left(T_{d}\right)$ of the original image.

\section{Integration of the three channels}

After summing the outputs of the three template sequences with a logical OR function $\left(T_{\mathrm{OR}}\right)$ and using a 'patch maker' template $\left(T_{b 4}\right)$ [Zarándy et al., 1994] to obtain filled patterns, we get the mouth patterns on the face and on the pepper (see Fig. 24, bottom of column B). The final step is the reproduction of the original colors from the input image $\left(T_{a b}\right)$.

\section{Color Processing}

In the retina, in the lateral geniculate nucleus and within the visual cortex of primates the color is coded by the activity of single and double color opponent cells [Kandel et al., 2000c]. The receptive field of single-opponent cells [see Fig. 26(a)] is characterized by a center-surround antagonism but the center and surround are stimulated by different light wavelengths (different sets of cones). Single opponent cells transmit information about both brightness and color. The receptive field of doubleopponent cells [Fig. 26(b)] is formed by singleopponent neurons, also of center-surround type, but double opponent cells are sensitive only to color contrast. They are found only in the primate primary visual cortex.

The template simulating the single-opponent cell has two layers. The input of the first layer is the monochromatic red map, while the second layer gets the green map [Roska et al., 1993c]. The result appears on the second layer. The CNN template is the following:

$B_{12}=\left[\begin{array}{lll}0 & 0 & 0 \\ 0 & 2 & 0 \\ 0 & 0 & 0\end{array}\right] \quad B_{22}=\left[\begin{array}{ccc}-0.25 & -0.25 & -0.25 \\ -0.25 & 0 & -0.25 \\ -0.25 & -0.25 & -0.25\end{array}\right]$

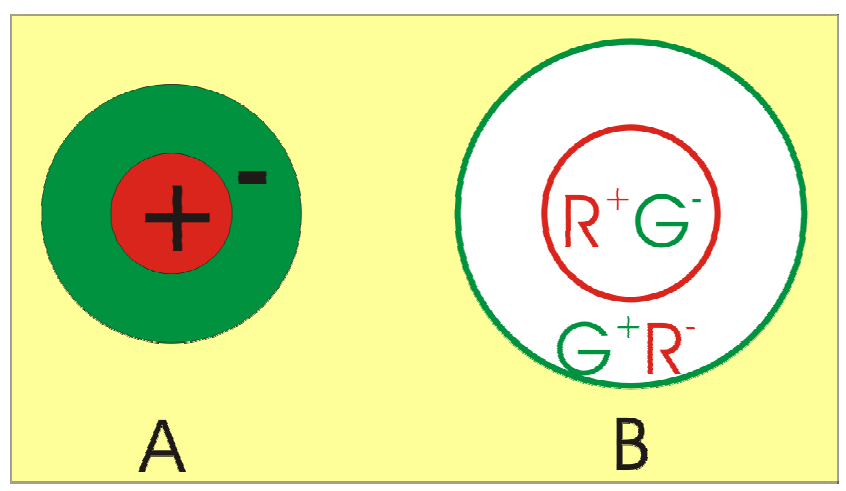

Fig. 26. Receptive fields of color processing single-opponent and double-opponent cells.

By swapping the layers we get the template generating the $\mathrm{G}+\mathrm{R}$ - single opponents. The outputs of the $\mathrm{R}+\mathrm{G}-$ and $\mathrm{G}+\mathrm{R}$ - layers provide the input for the first and the second layers of the double-opponent structure, respectively. The output appears on the second layer. The template is as follows:

$$
\begin{aligned}
B_{12} & =\left[\begin{array}{lllllll}
0 & 0 & 0 & 0 & 0 & 0 & 0 \\
0 & 0 & 0 & 0 & 0 & 0 & 0 \\
0 & 0 & 0 & 0 & 0 & 0 & 0 \\
0 & 0 & 0 & 2 & 0 & 0 & 0 \\
0 & 0 & 0 & 0 & 0 & 0 & 0 \\
0 & 0 & 0 & 0 & 0 & 0 & 0 \\
0 & 0 & 0 & 0 & 0 & 0 & 0
\end{array}\right] \\
B_{22} & =\left[\begin{array}{ccccccc}
0.02 & 0.02 & 0.02 & 0.02 & 0.02 & 0.02 & 0.02 \\
0.02 & 0 & 0 & 0 & 0 & 0 & 0.02 \\
0.02 & 0 & 0 & 0 & 0 & 0 & 0.02 \\
0.02 & 0 & 0 & 0 & 0 & 0 & 0.02 \\
0.02 & 0 & 0 & 0 & 0 & 0 & 0.02 \\
0.02 & 0 & 0 & 0 & 0 & 0 & 0.02 \\
0.02 & 0.02 & 0.02 & 0.02 & 0.02 & 0.02 & 0.02
\end{array}\right]
\end{aligned}
$$

Simulations (color image processing by $\mathrm{CNN}$ ):

Here some applications of the above described, single-opponent and double-opponent templates are shown. In Fig. 27 color input picture and the induced output picture are exhibited when using the single opponent template. Here the RFI-2 prototypes were applied.

In Fig. 28, the input picture is the same, but the output is a result of a double-opponent template. 

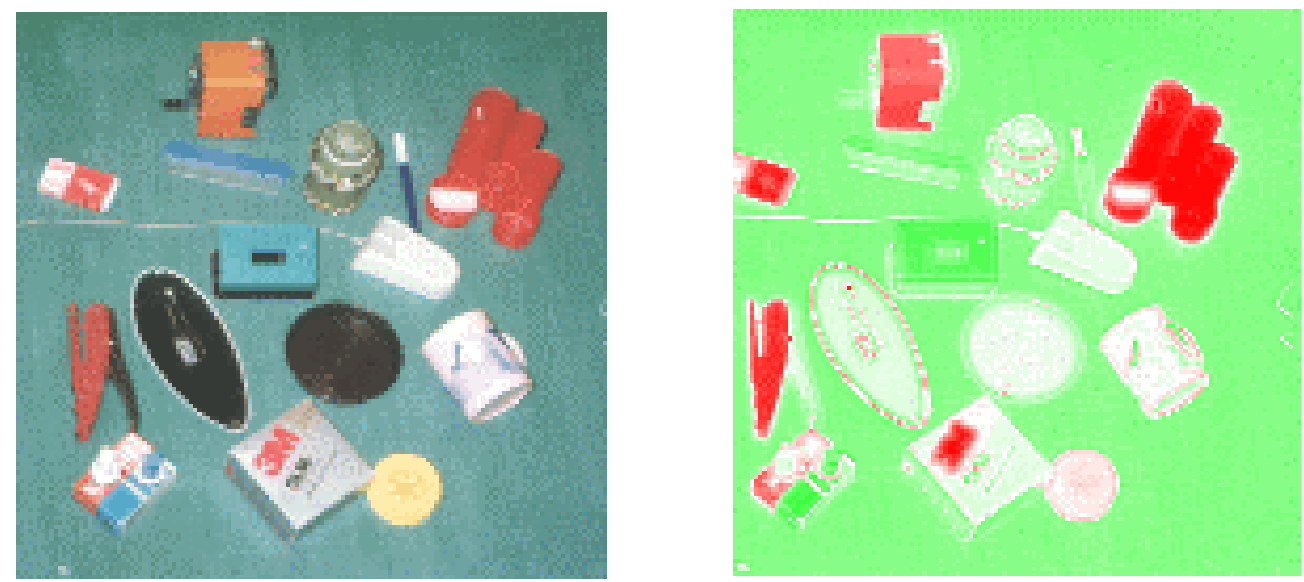

Fig. 27. Processing with a single-opponent template.
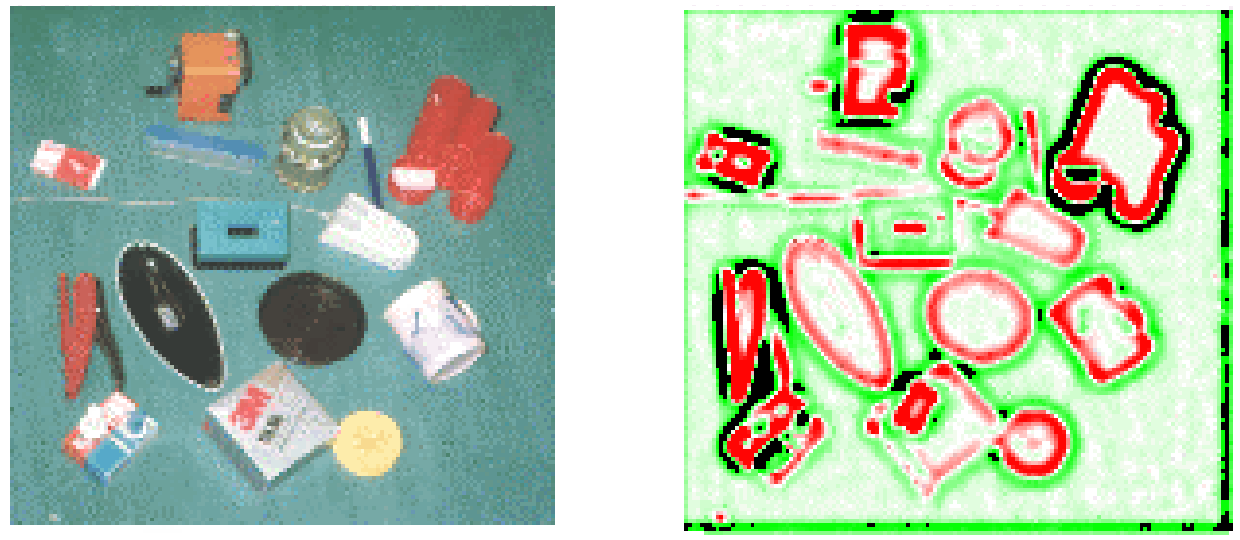

Fig. 28. Processing with a double-opponent template.

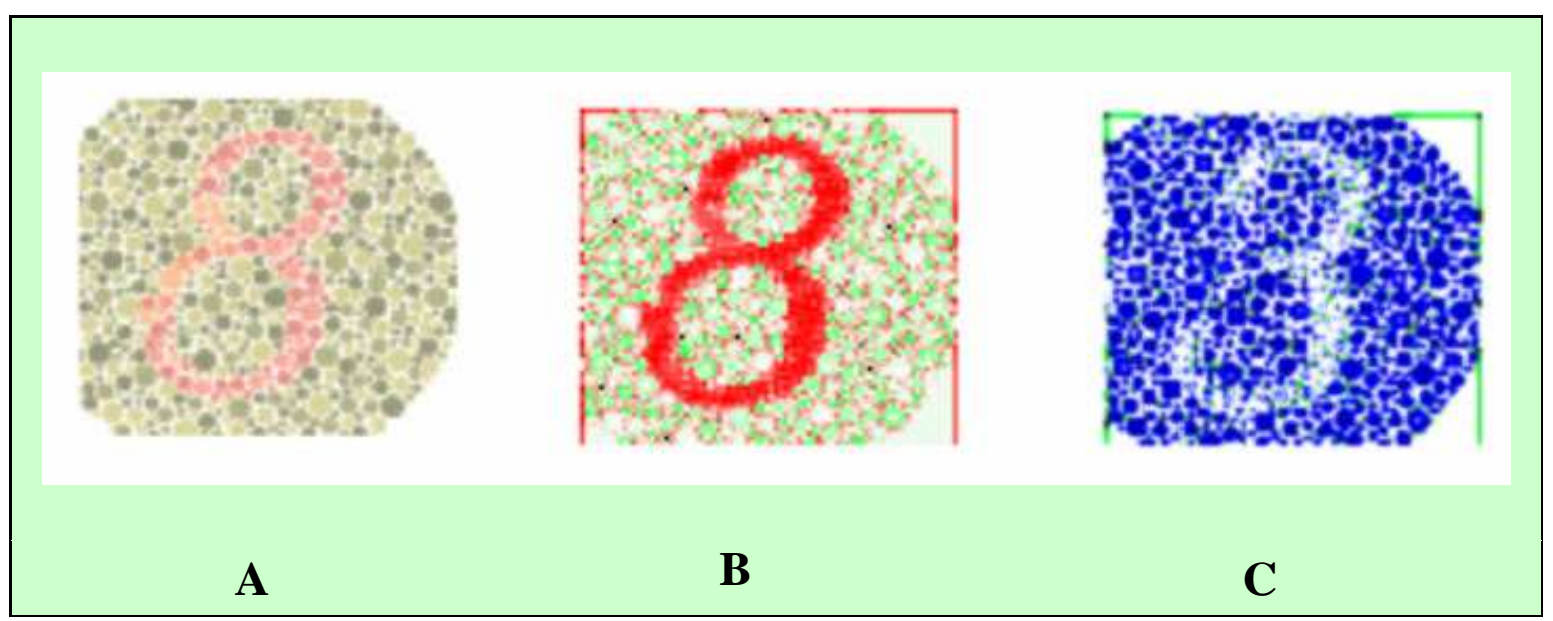

Fig. 29. Ishihara test processing: (A) input, (B) processing with a red-green single-opponent template, (C) processing with a yellow-blue single-opponent template. 


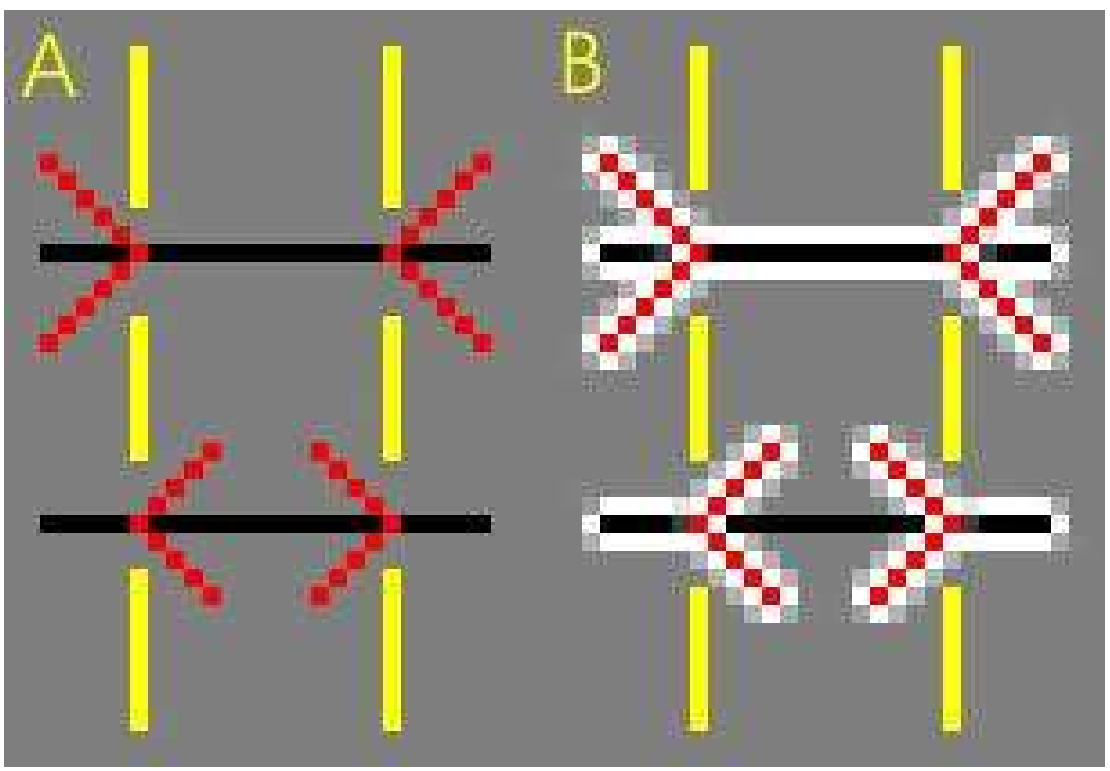

Fig. 30. (a) The Müller-Lyer illusion: the segment delimited by the arrows pointing toward each other looks longer than the one marked by the arrows directed away from each other. The one with the inward facing arrowheads looks shorter than the one with the outward facing tails. (b) An appropriate center-surround template operation on the image in (a) gives comparable results as the illusion effect. Two black pixels from the section between the outward facing tails are removed (turned to white) so it becomes shorter than the other one.

In Fig. 29 an application of single-opponent templates to an original Ishihara test image (used frequently in ophthalmology for the diagnosis of color blindness) is given. The first picture (a) is the Ishihara test image, the second (b) is the output resulting from a red-green $(\mathrm{R}+\mathrm{G}$ - $)$ single-opponent template (number 8 can be recognized) and the third (c) is the output after using a yellow-blue $(\mathrm{RG}+\mathrm{B}-)$ single-opponent template (number 3 pops out).

\section{Visual Illusions}

Generating visual illusions are essential tools of a psycho-physiologist in order to study the human visual system in a noninvasive way. We already know many strange effects without a well-established explanation. The authors designed some partly neuromorphical, partly phenomenological models as possible interpretations for a couple of illusions. More sophisticated cases can be found in [Zarándy et al., 1999]

\subsection{Müller-Lyer illusion}

The antagonistic center-surround organization can deceive the human visual system's length perception. As demonstrated in Fig. 30, the human ob- server incorrectly sees that arrows pointing toward each other delimit a longer interval than those directing away from each other. A simple antagonistic center-surround template (RF-2a prototype, Eq. (21)) can simulate the perceived phenomenon.

$$
A=0 \quad B=\left[\begin{array}{rrr}
-0.25 & -1.00 & -0.25 \\
-1.00 & 3.00 & -1.00 \\
-0.25 & -1.00 & -0.25
\end{array}\right] \quad z=0 ;
$$

\subsection{Herring grid}

Illusion: Gray patches appear at the intersections of a grid of black squares on a white background [see Fig. 31(a)].

CNN simulation: When applying a simple center-surround template to the above picture an error occurs simulating the illusion: bright patches appear in the middle of the black squares too [Fig. 31(b)]. The CNN retina model given in Sec. 3 simulates the illusion correctly [Fig. 31(c)].

\subsection{Zöllner illusion}

Figure 32 shows a distortion illusion. The lines are parallel, but the crossing lines cause them to appear to diverge.

To understand the phenomenon, one has to examine carefully, what kinds of effects the crossing 


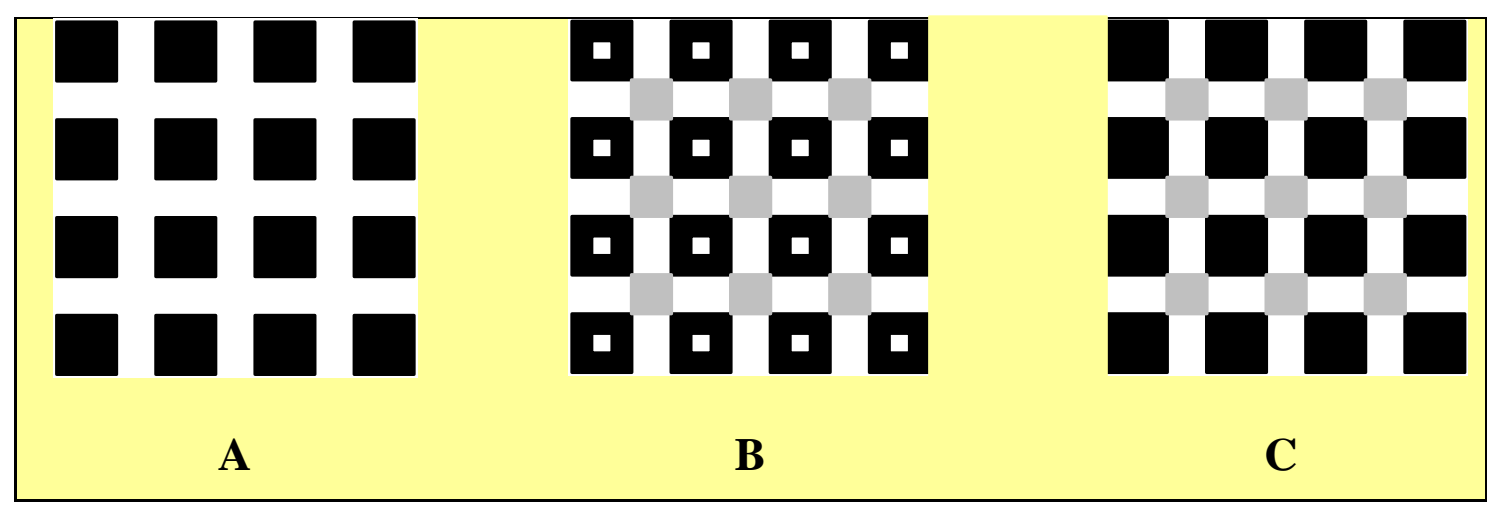

Fig. 31. (A) Herring grid illusion. Intersections of the white stripes seem to be darker than the stripes themselves. (B) Result of a simple center-surround template operation on picture (A). An unwanted effect is that within the black squares white patches pop up. (C) Processing the same image by the retina model described in Sec. 3 reproduces the illusion correctly.

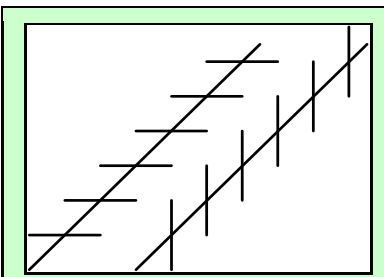

A

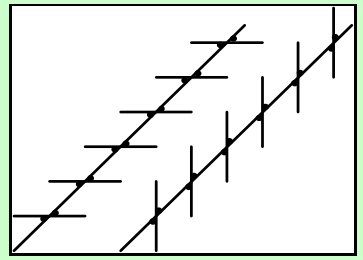

B

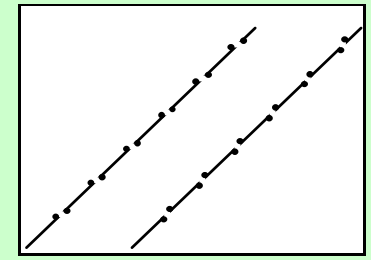

C

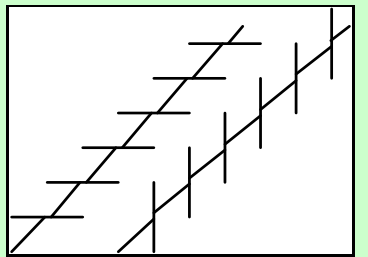

D

Fig. 32. (A) The original Zöllner illusion: the parallel lines, the crossing segments appear to diverge. (B) The magnified patches at the acute angles. (C) The cut sections with the patches. (D) The magnified distortion caused by the crossing lines.

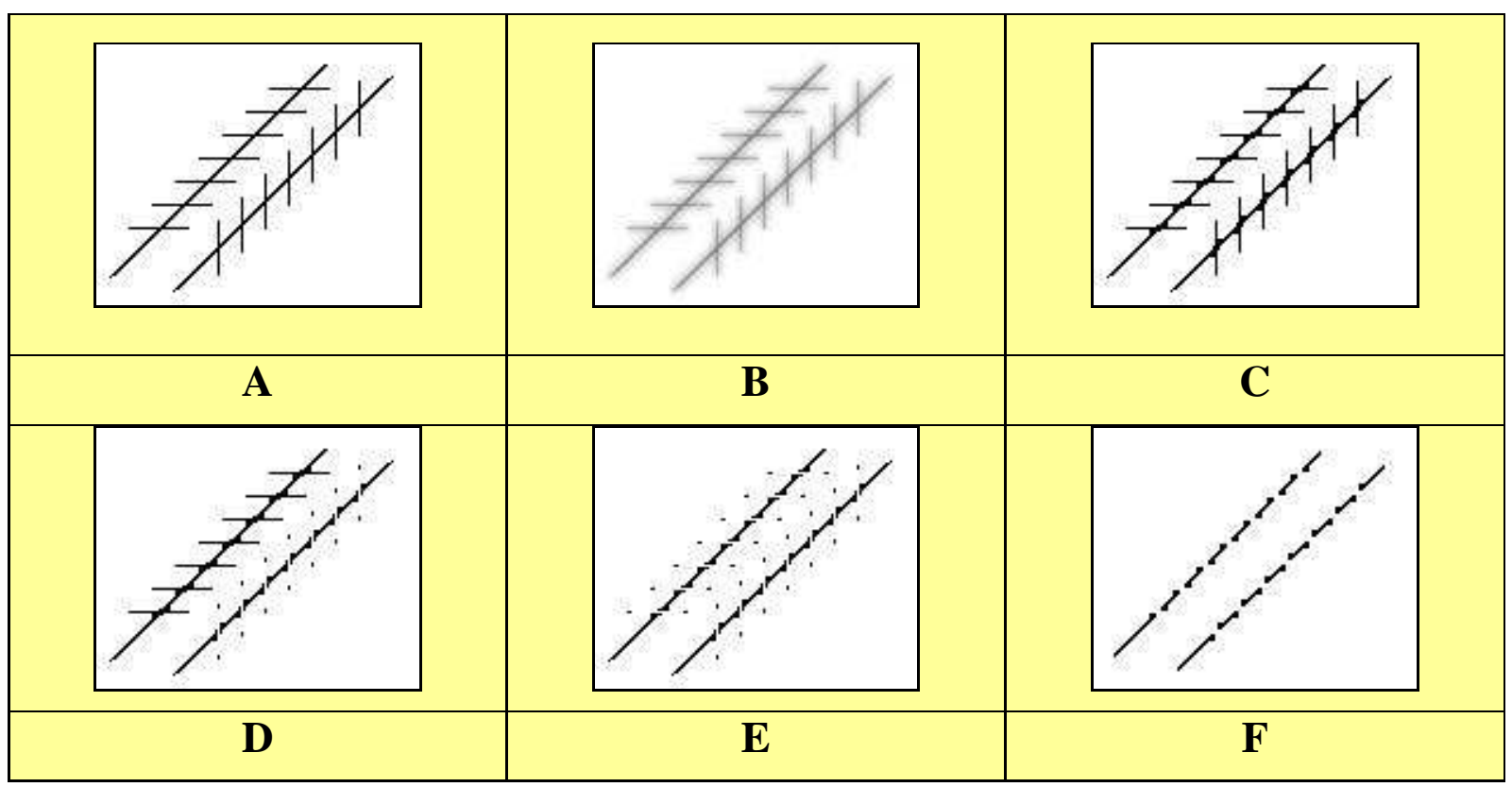

Fig. 33. (A) The original test image. (B) The blurred image. (C) The result of the threshold function. (D) Erasing the vertical crossing lines. (E) Removing the horizontal crossing lines. (F) The line segments with the patches. 
sections have on the two lines. The most important primary effect is that small black patches appear only at the acute angles but not at the obtuse angles. This can be seen even by the naked eye [Fig. 32(a)]. The small black patches are exaggerated in Fig. 32(b). We consider, that in a higher level of our visual system the crossing lines are erased, but the patches still remain at the end of the sections [Fig. 32(c)].

The patches at the end of the line segments fool the brain when it computes the orientation: the ends of the segments seem to be tilted towards the patches, so they appear being rotated. In Fig. 32(d) the effect is overemphasized, which illustrates what the human eye sees.

A structural-strategy model is designed for this illusion. The appearance of the patches is derived from the structure of the retina, while the rotation effect is explained by the behavior of the orientation selective cells in the cortex [Kandel et al., 2000c].
The appearance of the patches is the result of a blurring effect used in subcortical neuromodels [Roska et al., 1993b]. This can be modeled by the following feedforward template:

$$
\begin{aligned}
A & =[0], \\
B & =\left[\begin{array}{lllll}
0.023 & 0.023 & 0.023 & 0.023 & 0.023 \\
0.023 & 0.053 & 0.055 & 0.053 & 0.023 \\
0.023 & 0.055 & 0.2 & 0.055 & 0.023 \\
0.023 & 0.053 & 0.055 & 0.053 & 0.023 \\
0.023 & 0.023 & 0.023 & 0.023 & 0.023
\end{array}\right], \\
z & =0
\end{aligned}
$$

The effect of the template can be seen in Figs. 33(a) and $33(\mathrm{~b})$.

The gray-scale result of this template is thresholded to get a black-and-white image again [Figs. 33(b) and 33(c)].

The following three templates remove the crossing lines [see Figs. 33(c)-33(f)]:

$$
\begin{aligned}
& A=[2], \quad B=\left[\begin{array}{lllllll}
-0.2 & -0.2 & -0.2 & 1 & -0.2 & -0.2 & -0.2
\end{array}\right]^{T}, \quad z=-1.3 \\
& A=[2], \quad B=\left[\begin{array}{lllllll}
-0.2 & -0.2 & -0.2 & 1 & -0.2 & -0.2 & -0.2
\end{array}\right], \quad z=-1.3
\end{aligned}
$$

\begin{tabular}{|c|c|c|c|c|c|c|c|c|c|c|c|c|c|c|}
\hline & & & & & & \multirow{7}{*}{$A=[0], B=$} & {$[-0.095$} & -0.048 & 0.071 & 0.14 & 0.071 & -0.048 & -0.095 & \multirow{7}{*}{$z=0$} \\
\hline- & -1. & ++ & ++ & - & - & & -0.095 & -0.048 & 0.071 & 0.14 & 0.071 & -0.048 & -0.095 & \\
\hline- & - & $\begin{array}{c}+ \\
++ \\
+t\end{array}$ & + & - & - & & -0.095 & -0.048 & 0.071 & 0.14 & 0.071 & -0.048 & -0.095 & \\
\hline- & - & ++ & ++ & - & - & & -0.095 & -0.048 & 0.071 & 0.14 & 0.071 & -0.048 & -0.095 & \\
\hline- & \begin{tabular}{|l}
- \\
-
\end{tabular} & $\begin{array}{l}+ \\
+\end{array}$ & + & - & - & & -0.095 & -0.048 & 0.071 & 0.14 & 0.071 & -0.048 & -0.095 & \\
\hline- & - & +- & ++ & - & - & & -0.095 & -0.048 & 0.071 & 0.14 & 0.071 & -0.048 & -0.095 & \\
\hline & & & & & & & -0.095 & -0.048 & 0.071 & 0.14 & 0.071 & -0.048 & -0.095 & \\
\hline
\end{tabular}

Fig. 34. The receptive field organization of the modeled orientation selective cortical cell type.

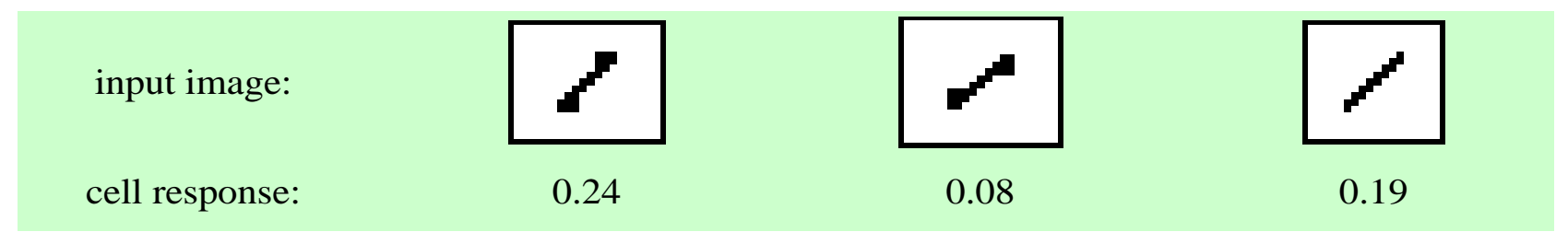

Fig. 35. The images in the receptive field of the modeled orientation selective cells, and their response level (output range can vary between $(-1,+1))$. 


$$
A=\left[\begin{array}{lll}
0 & 1 & 0 \\
1 & 2 & 1 \\
0 & 1 & 0
\end{array}\right], \quad B=[0], \quad z=0
$$

For computing the orientation, there are orientation selective cells in the cortex. The receptive field organization and the corresponding template of a vertically oriented, orientation selective cortical cell type can be seen in Fig. 34 .

The inputs are shown in Fig. 35. The orientation of the bar in the receptive field of a cell determines its output value. The modeled orientation selective cell was tested with three different images. Two of them were line segments with patches at the ends [from Fig. 33(f)], and the third was a same sized and oriented section without patches. This third one is considered as a reference image (Fig. 35). The values of the responses are also indicated in Fig. 35. According to the receptive field organization of the modeled cell type, the bigger the slope of the line in the receptive field, the greater the response. Note that the responses are not proportional to the deviations. It is not surprising, because the way the cell computes the orientation is nonlinear.

\section{Two-Point Discrimination in Tactile Sensing}

In addition to the density of the mechanoreceptors in the skin, two-point discrimination is known to depend on the overlap and the size of their central neural representation [Kandel et al., 2000a].

The simplest example for spatial discrimination is the distinguishing of two closely placed point stimuli. When the skin is stimulated at one point several receptors are also activated in the neighborhood of the stimuli. These impulses travel through different structures of the somatosensory system to the cortex. The activated areas will overlap and the two activity peaks could be merged. However, in the different relay stations neurons conveying the stimulus activate only a set of the target cells where the activation of inhibitory interneurons restricts the population of these active neurons. Moving from the center to the edge of the active region, the activity level gradually decreases, while the active zone is surrounded by an inhibited ring of neurons [Kandel et al., 2000a]. Inhibition increases the contrast of the stimulus. This kind of lateral inhibition presumably plays an important role in a better spatial discrimination, and could be the site for practice driven plasticity.

A simple CNN implementation based on divergent input and feedback inhibition to different layers (see Fig. 36) representing the different structures of the somatosensory system highlights the key points of the model explained above.

The model follows the structure indicated in Fig. 36. The CNN implementation integrates the receptor layer and the first processing layer. The A and B templates are two discretized Gauss density functions which are sampled in the $[-2,2]$ interval integer points and have the following form:

$$
\begin{aligned}
& \begin{array}{c}
\mathbf{A}: \\
\sigma=1.6
\end{array} \quad a_{i j}=-3 \frac{1}{\sum_{i j} \exp \left(-\frac{x^{2}+y^{2}}{2 \sigma^{2}}\right)} \exp \left(-\frac{x^{2}+y^{2}}{2 \sigma^{2}}\right) \\
& \left.\begin{array}{c}
\mathbf{B}: \\
\sum_{i j} \exp \left(-\frac{x^{2}+y^{2}}{2 \sigma^{2}}\right)
\end{array} \quad b_{i j}=4 \frac{x^{2}+y^{2}}{2 \sigma^{2}}\right) \\
& z=0
\end{aligned}
$$

where $\sigma$ is the deviation, $i, j$ are integers, $i, j \in$ $[-2,2]$. The 0,0 values of the $i, j$ indices stand for the central element of the A and B matrices.

The shape of A and B templates follow the property of the somatosensory system, that the inhibition is mostly covered by the more effective excitation. The effect of the $\mathbf{A}$ template becomes less important in the center of the activity region compared to the effect of $\mathbf{B}$ (see Fig. 39). Lighter pixels indicate greater activity.

The results can be seen in Figs. 37 and 38. In Fig. 37 the activity region (white pixels) and the surrounding inhibited region of neurons (dark 


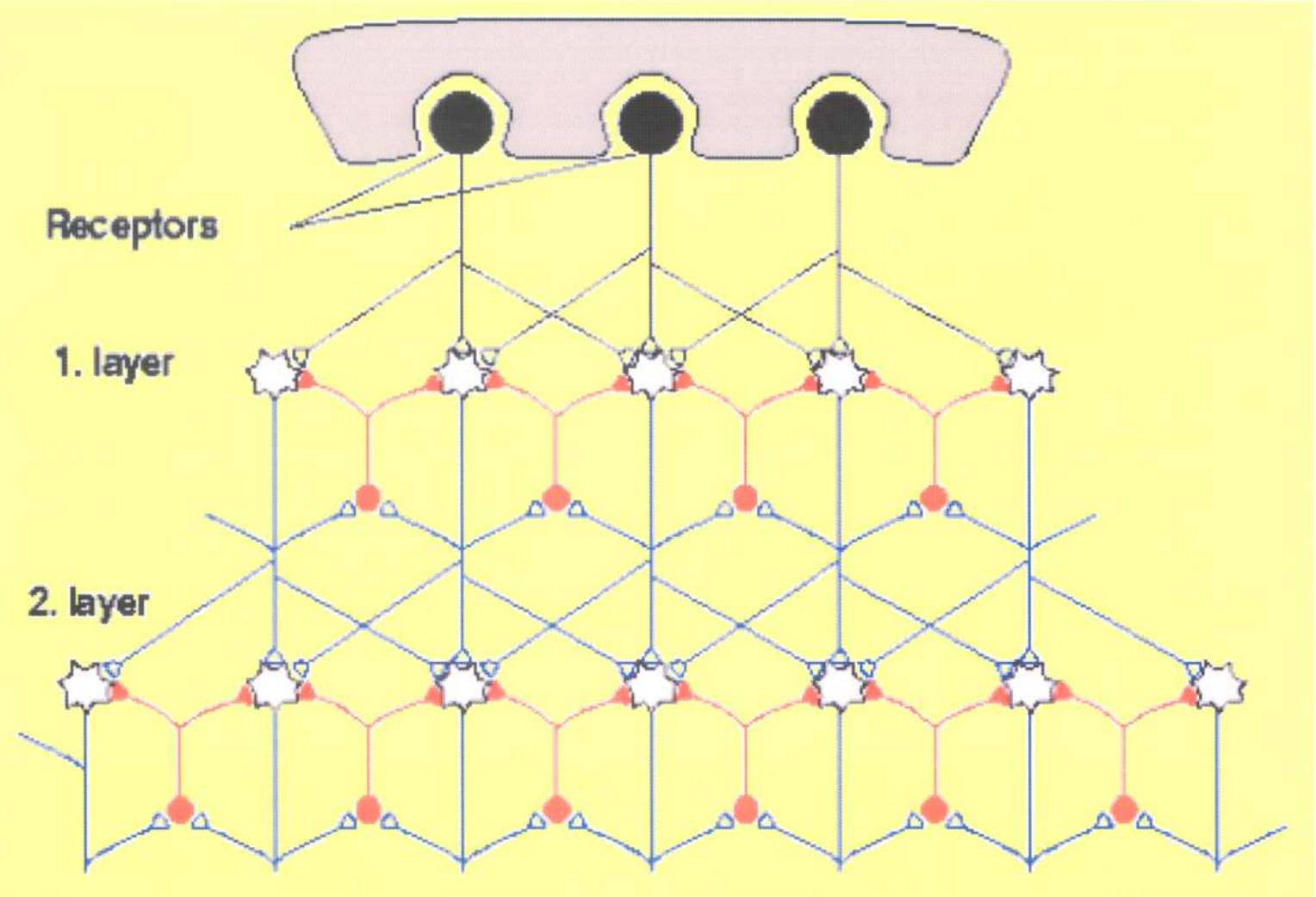

Fig. 36. Schematic view of the network of the modeled somatosensory system. The inhibitory interneurons between the layers marked with red solid circles feedback the output of the cells with some delay.

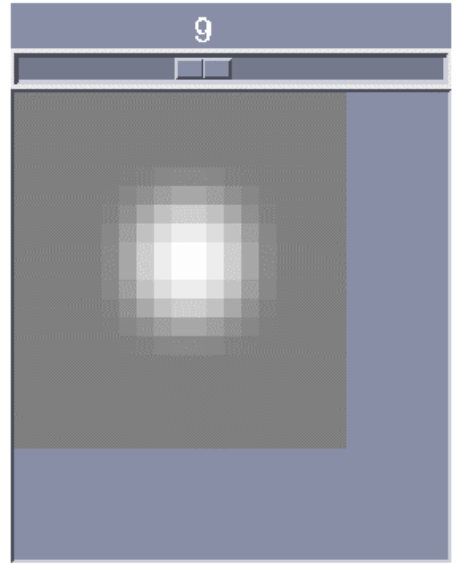

input

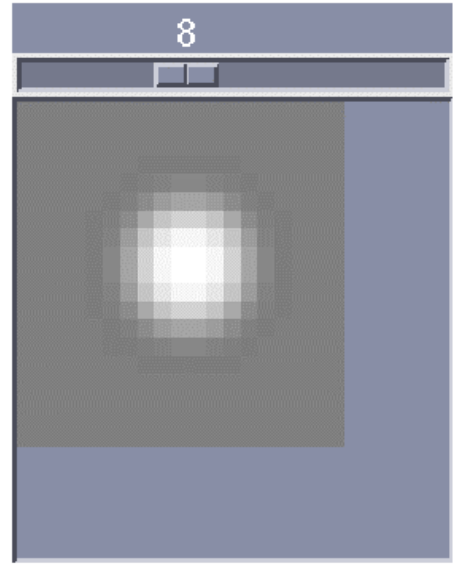

output of the 1st layer

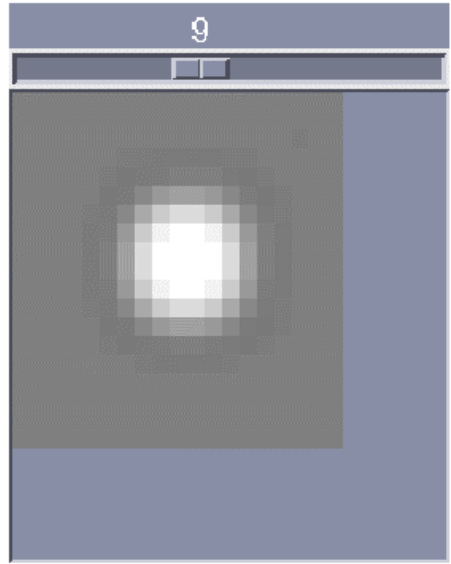

output of 2nd layer

Fig. 37. The effect of feedback inhibition when only one, spot shaped stimulus is present (simulated result). The inhibited region around the patch on the output of the second layer is darker and broader than on the output of the first layer. 


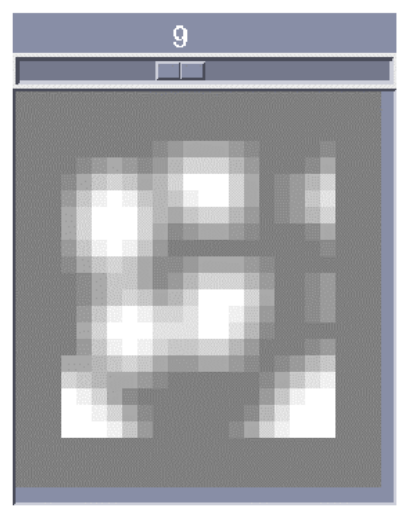

input

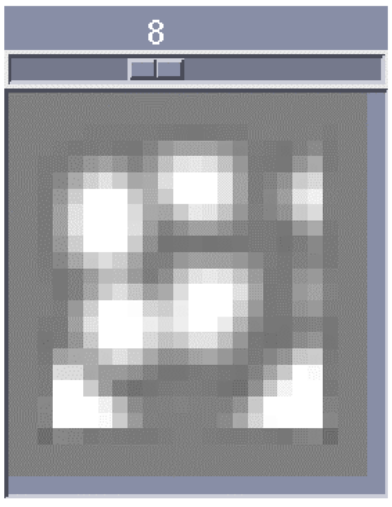

output of

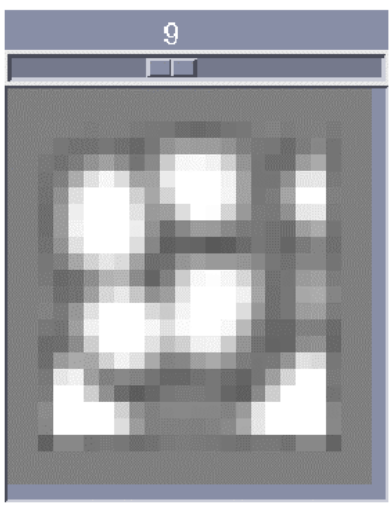

output of the 2nd layer

Fig. 38. The effect of lateral inhibition. Simulation is based on the proposed model. The patches representing the stimuli are gradually separated on each layer, and the gaps are darkened even in those areas where the stimuli were close to each other. The contrast of the patches increased.

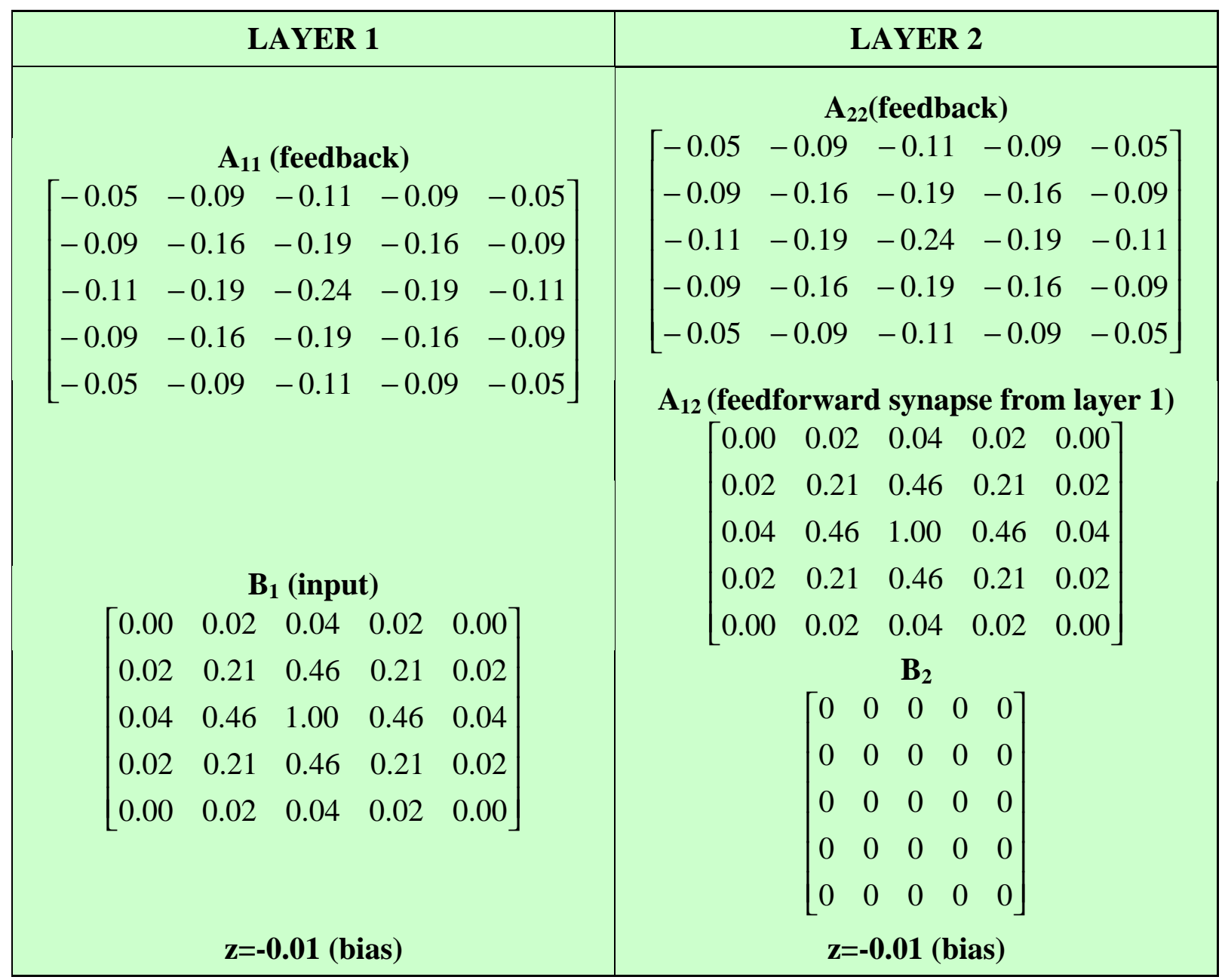

Fig. 39. Templates of the 2-layer CNN modeling the somatosensory processing. 
gray pixels) can be seen. In Fig. 38 closely located patches represent the stimuli. In the outputs of the layers a dark (inhibited) region can be observed among the patches and the peaks are gradually discriminated.

\section{Hyperacuity in Time in the Auditory System}

The barn owl can catch its pray in total darkness relying solely on acoustic signals. It can localize sound within $1-2^{\circ}$ in azimuth and elevation and can detect interaural time differences as little as some tens of microseconds. The neural mechanism underlying this fascinating ability has been investigated for decades [Takahashi \& Konishi, 1986; Konishi et al., 1988; Konishi, 1991, 1992]. The two main problems we have in sound localization:

- How can an owl detect interaural time differences as little as some tens of microseconds while a single action potential persists considerably longer, on the order of 1000 microseconds at least?

- How can an owl resolve phase ambiguity which comes from the fact, that time is measured by the phase of the input signals? That is, higher order neurons receive phase-locked spikes from the left and right ears, and these neurons, therefore, can give maximal response not only to one but to several interaural time differences.

We are going to show an answer to the first question, which involves a type of hyperacuity (in time): the sound localization system can mark shorter delays of time arrivals of sound than the duration of an impulse, which indicates the time arrival. A more exhaustive description of the model can be found in [Lotz et al., 1999].

Signals from the left and right ears pass through the basilar membranes and nucleus magnocellularis (NM) and converge in the nucleus laminaris (NL). There is hard evidence that interaural time difference (ITD) is mapped into neural place coding in the NL via coincidence detection of signals arriving from the left and right ears. The model — according to which the place of the neuron with maximum response specifies the corresponding ITD - was proposed by Jeffress [1948] (see Fig. 40). The accuracy of this model was verified by the experiments of Konishi and his colleagues [1988, 1991; Wagner et al., 1987]. Their findings indicate that the magnocellular (NM) afferents work as delay lines, and the NL neurons work as coincidence detectors [Carr \& Konishi, 1990].

In the CNN model spike duration is much greater than the time an action-potential requires to travel from one cell to the other. The velocity of spikes on the axon of a magnocellular cell is estimated to be $3-5 \mathrm{~m} / \mathrm{s}$ [Takahashi \& Konishi, 1986; Konishi et al., 1988] and the NL neurons have large cell bodies $(30-40 \mu \mathrm{m})$. If we take the velocity of spikes $4 \mathrm{~m} / \mathrm{s}$ and the distance of $2 \mathrm{NL}$ neurons $100 \mu \mathrm{m}$, then the time a signal travels between two neighboring neurons is $25 \mu \mathrm{sec}$. The duration of a

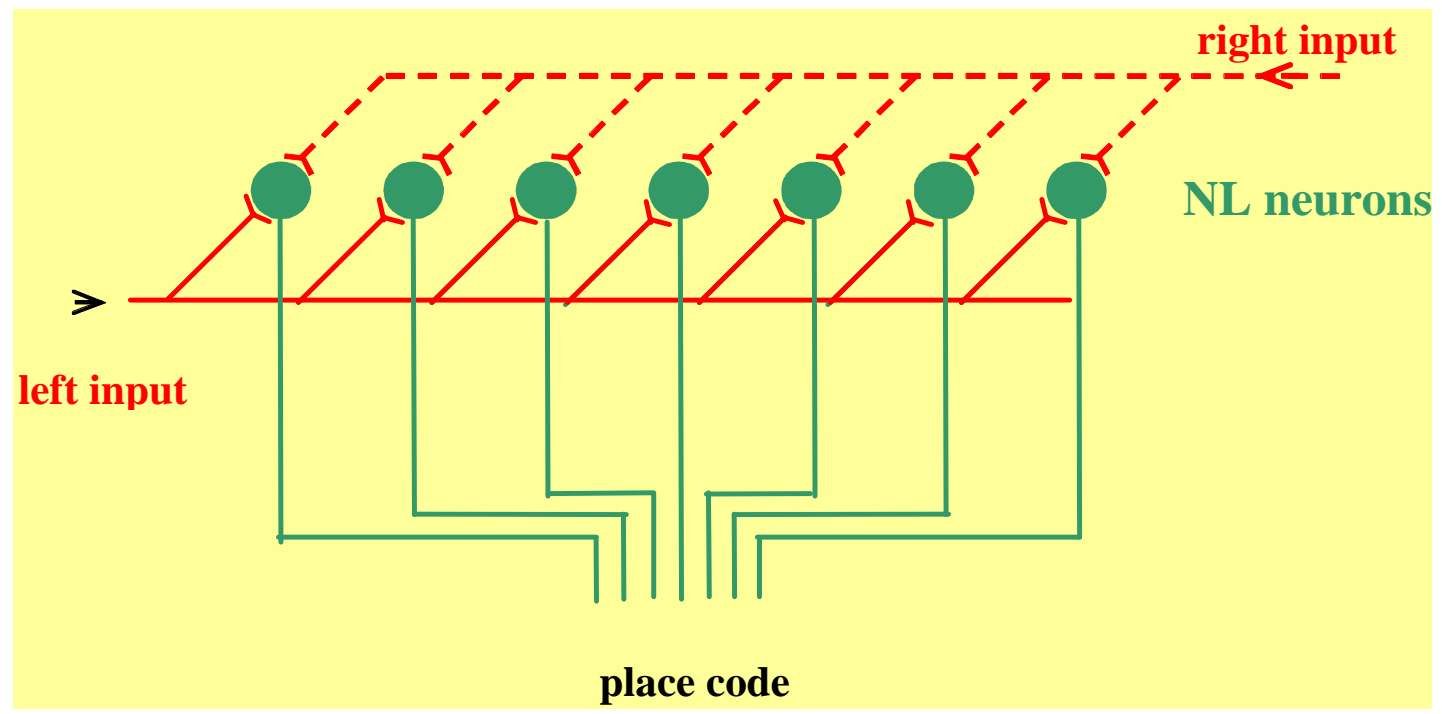

Fig. 40. The Jeffress model for encoding interaural time differences. 


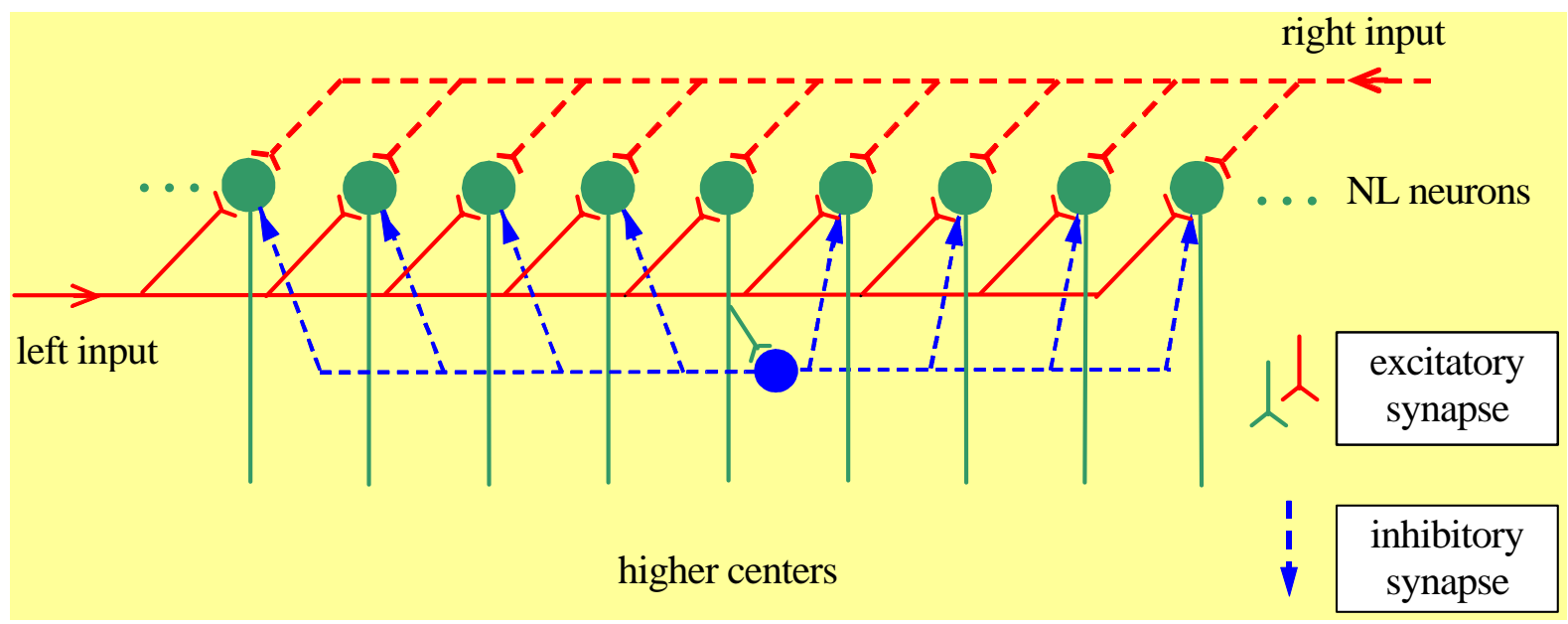

Fig. 41. The Jeffress model completed by lateral inhibition. Each neuron synapses on an inhibitory interneuron (for the sake of simplicity only one is indicated in the figure) which inhibits the neighboring cells.

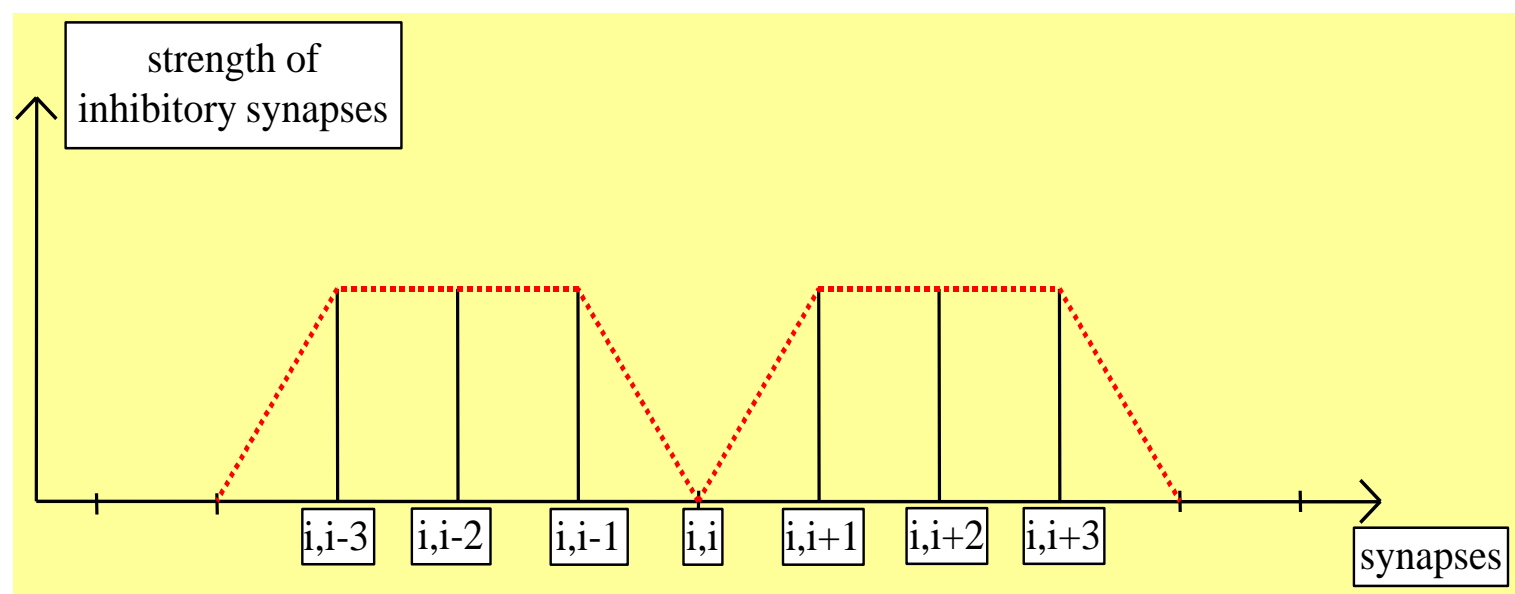

Fig. 42. Spatial distribution of inhibitory synaptic strengths.

real spike is about 2 milliseconds. It is also evident that a spike has no distinct peak along the line of NL neurons. The model, however, shows very good results in coincidence detection.

The original delay line-coincidence detector model (see Fig. 40) was completed with lateral inhibition (via interneurons) between the neighboring cells to study the effects of this inhibition on the neuron's ability to detect coincidence between the input signals [Fujita \& Konishi, 1991]. The new structure is shown in Fig. 41.

In this structure each NL neuron synapses on an inhibitory interneuron (for simplicity only one is indicated in Fig. 41), which inhibits the neighboring neurons (taking neighborhood 3). The number of modeled NL neurons in the line is 15 in accordance with the neuromorphological data. The CNN equivalent of the architecture consists of 4 layers, where each layer is a row of 15 cells. The specific templates are given in Fig. 44. For spike generation VCCS type connections are also required (see Sec. 2).

As neurons receive inhibitory signals from several sources, the net current — which determines the amplitude of inhibition - originating from the inhibitory synapses is:

$$
I_{i}=\left(V_{m}-E_{\mathrm{syn}}\right) \sum_{j=-3}^{3} k_{i j} g_{\mathrm{syn}}\left(V_{m}-E_{\mathrm{syn}}\right)
$$


Cell 5

Cell 6
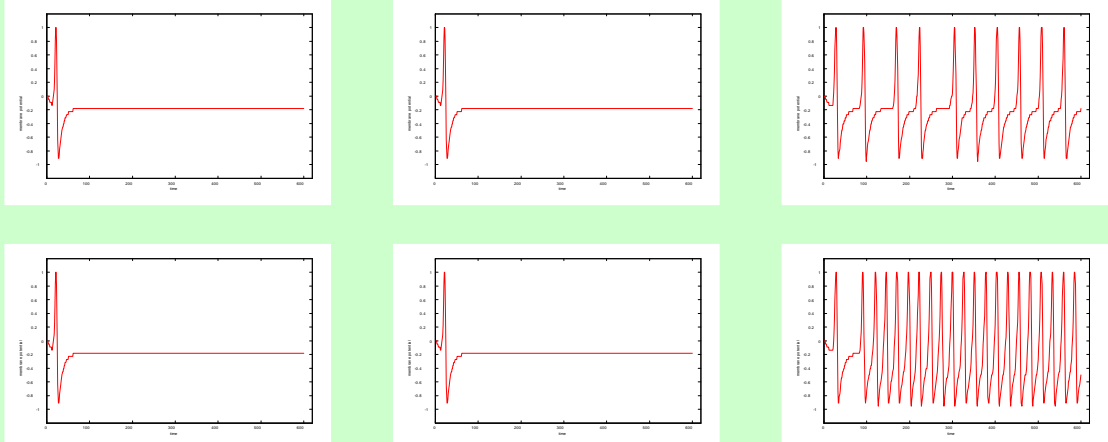

Cell 7
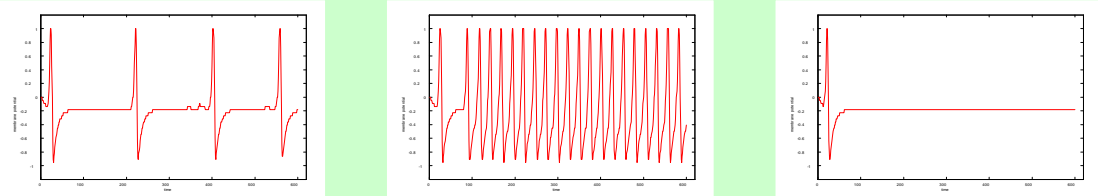

Cell 8
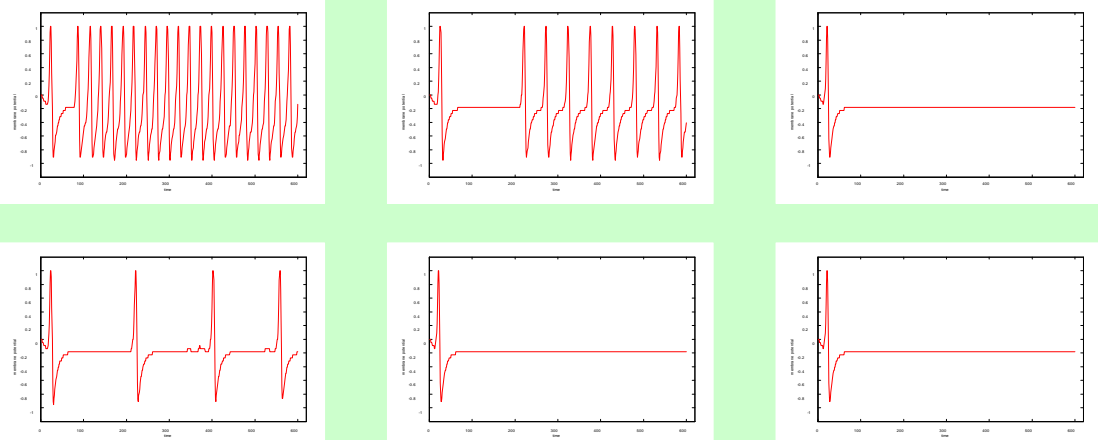

Cell 9

Cell 10
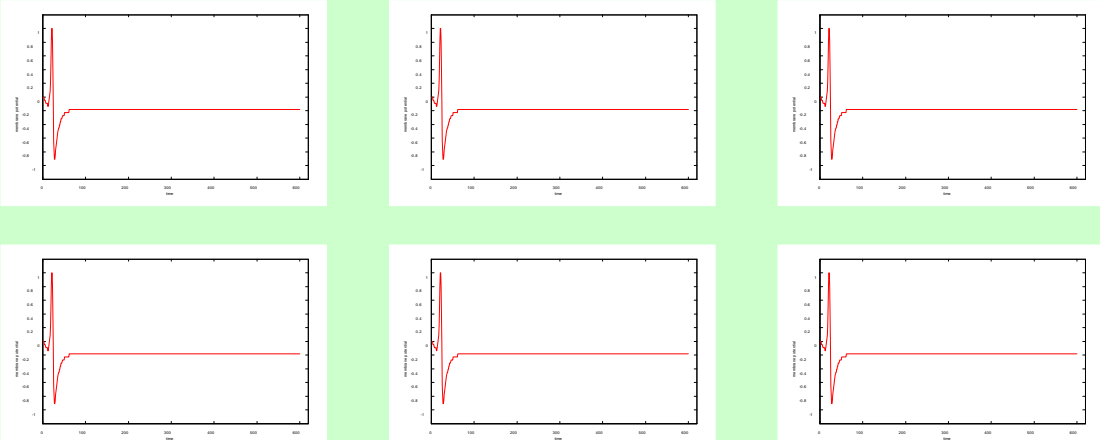

ITD

$$
\Delta \mathrm{t}=0
$$

$\Delta \mathrm{t}=30 \mu_{\mathrm{s}}$

$\Delta \mathrm{t}=60 \mu \mathrm{s}$

(a)

(b)

(c)

Fig. 43. Responses of seven cells for three different values of interaural time difference (ITD).

where $k_{i j}$ is the strength of the synapse between the $i$ th and $j$ th neurons, $E_{\text {syn }}$ is the equilibrium potential characterizing the inhibitory synapse, $\mathrm{V}_{m}$ stands for the membrane potential, $g_{\mathrm{syn}}$ is the synaptic conductance function. The different synap- tic conductance functions used here can be seen in Fig. 45.

The best coincidence detection was achieved when the spatial distribution of the inhibitory connection strengths was set as displayed in Fig. 42. 


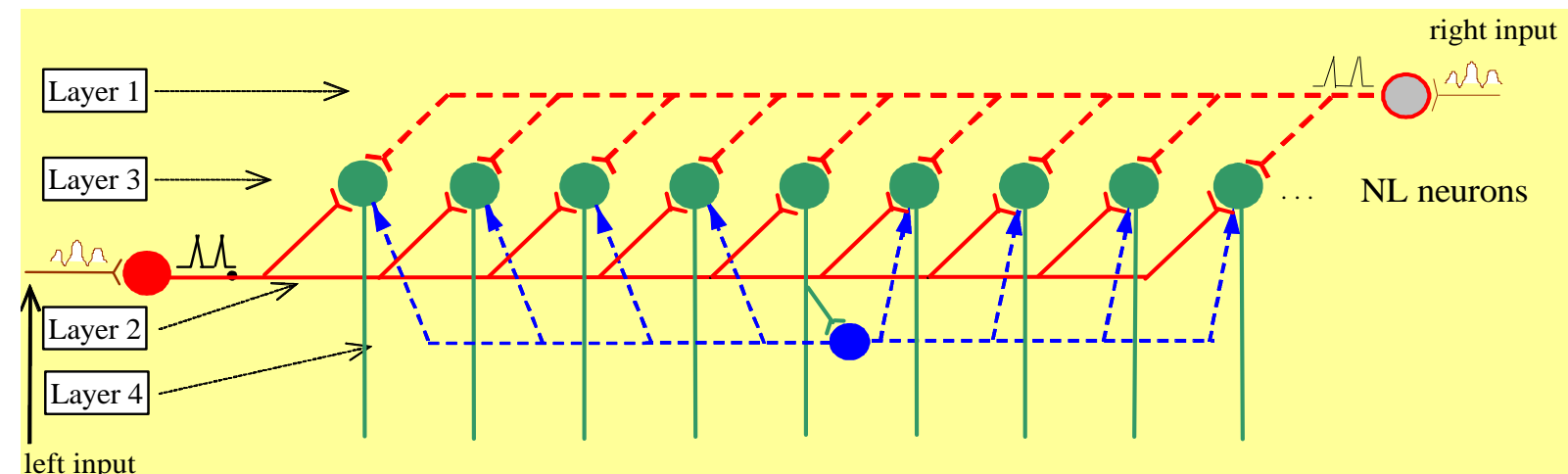

higher centers

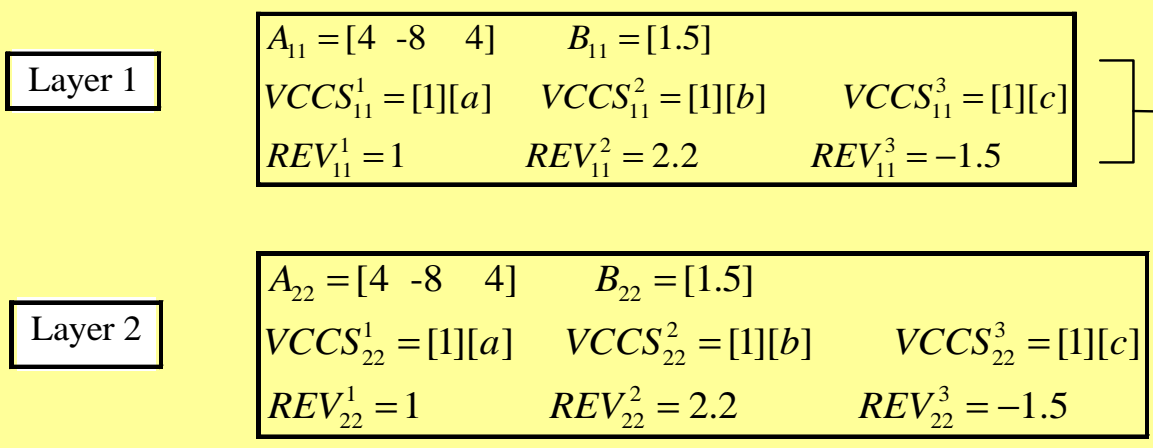

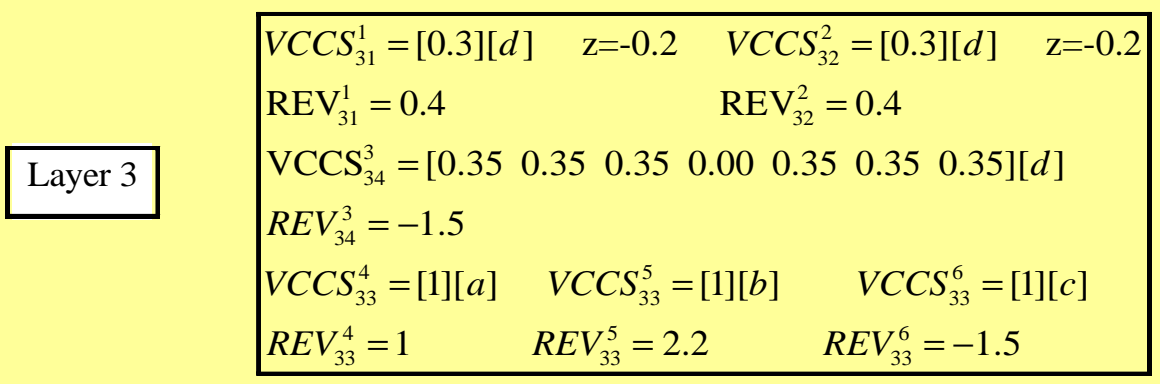

Layer 4

\begin{tabular}{|lcc|}
$V C C S_{43}^{1}=[0.6][d]$ & $R E V_{43}^{1}=0.5$ & \\
$V C C S_{44}^{2}=[1][a]$ & $V C C S_{44}^{3}=[1][b]$ & $V C C S_{44}^{4}=[1][c]$ \\
$R E V_{44}^{2}=1$ & $R E V_{44}^{3}=2.2$ & $R E V_{44}^{4}=-1.5$ \\
\hline
\end{tabular}

Fig. 44. The coincidence detector circuit. VCCS: Voltage controlled current sources. REV: Reversal (or equilibrium) potential. (a-d) Synaptic conductance functions (see Fig. 45). 


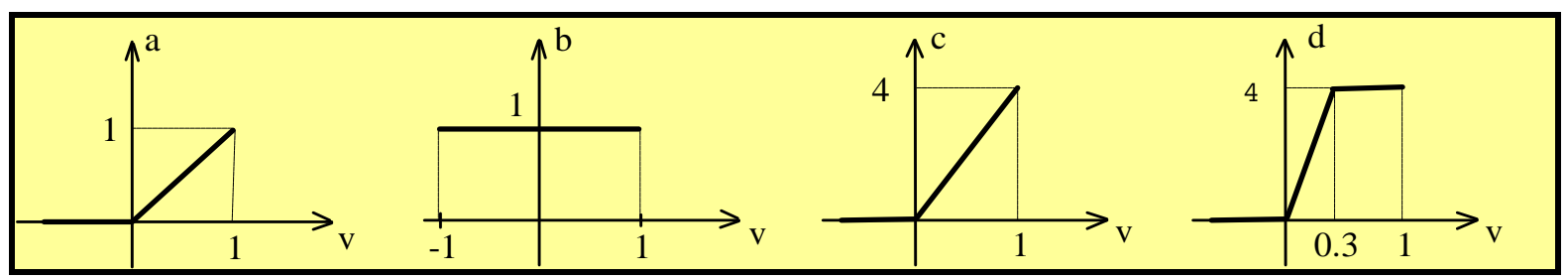

Fig. 45. Different synaptic conductance functions used in the model.

Self-inhibition must be little or zero to obtain good results.

The effects of the interneurons have also been examined on the system: the increasing inhibition gradually dissolves the ambiguity in coincidence detection, so it is very likely that inhibition plays a crucial role in this system.

In a simulation with 15 neurons the activity of seven cells was observed while the arrival time difference of the left and right input signals was changed. The inhibition was constant during this experiment $(k=0.4)$. Figure $43(\mathrm{a})$ shows the responses of the cells when the time difference is 0 . Coincidence is detected by Cell 8, which responded most vigorously. Delaying one of the input signals by $30 \mu \mathrm{s}$, Cell 7 detected the coincidence [see Fig. 43(b)]. Further delaying the same input by the same period, Cell 6 showed the strongest response [Fig. 43(c)] etc.

\section{Conclusion}

Many different sensory modalities can be modeled via the CNN paradigm. The key common notion is the combination of different layers and receptive fields. Since this is "prototype" architecture, as a receptive field "calculus" its programmable physical implementation via the CNN-UM chips and other systems lead to practical applications.

\section{Acknowledgments}

The support of the Office of Naval Research USA (ONR) grant no. N0001498-1-0052, the OTKA grant no. T026555, and the Hungarian Academy of Sciences are acknowledged.

\section{References}

Bálya, D., Roska, B., Roska, T. \& Werblin, F. S. [2002] "A CNN framework for modeling parallel processing in a mammalian retina," Int. J. Circuit Th. Appl. 30, 363-393.
Bálya, D., Petrás, I. \& Roska, T. [2004] "Implementing the multi-layer CNN retinal model on the complexcell stored program 2-layer CNN universal machine chip prototype," Int. J. Bifurcation and Chaos 14, 427-451.

Carr, C. E. \& Konishi, M. [1990] "A circuit for detection of interaural time differences in the brain stem of the barn owl," J. Neurosci. 10, 3227-3246.

Chua, L. O. \& Yang, L. [1988] "Cellular neural networks: Theory and application," IEEE Trans. Circuits Syst. 35, 1257-1290.

Chua, L. O. \& Roska, T. [1993] "The CNN paradigm," IEEE. Trans. Circuits Syst. 40, 147-156.

Chua, L. O. [1997] "CNN: A vision of complexity," Int. J. Bifurcation and Chaos 7, 2219-2425.

Chua, L. O. \& Roska, T. [2002] Cellular Neural Networks and Visual Computing (Cambridge University Press, Cambridge).

Dowling, J. E. [1987] The Retina: An Approachable Part of the Brain (Harward University Press, London)

Fujita, I. \& Konishi, M. [1991] "The role of GABAergic inhibition in processing of interaural time difference in the owl's auditory system," J. Neurosci. 11, 722-739.

Fujita, I., Tanaka, K., Ito, M. \& Cheng, K. [1992] "Columns for visual features of objects in monkey inferotemporal cortex," Nature 360, 343-346.

Hámori, J., Pasik, T., Pasik, P. \& Szentágothai, J. [1974] "Triadic synaptic arrangements and their possible significance in the lateral geniculate nucleus of the monkey," Brain Res. 80, 379-393.

Hámori, J. \& Roska, T. (eds.) [2000] "Receptive field atlas of the retinotopic visual pathway and some other sensory organs using dynamic cellular neural network models," Report DNS-8-2000, Budapest.

Http://INSIGHT.MED.EDU/WEBVISION

Http://www.illusionworks.com

Hubel, D. \& Wiesel, T. [1962] "Receptive fields, binocular interaction and functional architecture in the cat's visual cortex," J. Physiol. 160, 106-154.

Jeffres, L. A. [1948] "A place theory of sound localization," J. Comp. Physiol. Psychol. 41, 35-39.

Johnston, D. \& Wu, M.-S. [1995] Cellular Neurophysiology (MIT Press, London).

Kandel, E. R., Schwartz, J. H. \& Jessel, T. M. [2000a] Principles of Neural Science, 4th edition 
(McGraw-Hill/Appleton \& Lange, NY), Chap. 23, pp. 451-471.

Kandel, E. R., Schwartz, J. H. \& Jessel, T. M. [2000b] Principles of Neural Science, 4th edition (McGrawHill/Appleton \& Lange, NY), Chap. 26, pp. 507-522.

Kandel, E. R., Schwartz, J. H. \& Jessel, T. M. [2000c] Principles of Neural Science, 4th edition (McGrawHill/Appleton \& Lange, NY), Chap. 27, pp. 523-547.

Konishi, M., Takahashi, T. T., Wagner, H., Sullivan, W. E. \& Carr, C. E. [1988] "Neurophysiological and anatomical substrates of sound localization in the owl," in Auditory Function: Neurobiological Bases of Hearing, eds. Edelman, G. M., Gall, W. E. \& Cowan, W. M. (John Wiley).

Konishi, M. [1991] "Deciphering the brain's codes," Neural Comput. 3, 1-18.

Konishi, M. [1992] "The neural algorithm for sound localization in the owl," in The Harvey Lectures, Series 86, 47-64.

Lábos, E., Pasik, P., Hámori, J. \& Nógrádi, E. [1990] "On the dynamics of triadic arrangements: Computer experiments on formal neural nets of chaotic units," J. Hirnforschung 31, 715-722.

Linsenmeier, R. A., Frishman, L. J., Jakiela, H. G. \& Enroth-Cugell, C. [1982] "Receptive field properties of $x$ and $y$ cells in the cat retina derived from contrast sensitivity measurements," Vis. Res. 22, 1173-1183.

Lotz, K., Zarándy, Á., Roska, T. \& Hámori, J. [1995] "An analogic phenomenological CNN algorithm to model the mouth detection task of the inferotemporal cortex discovered by I. Fujita," Proc. Int. Symp. Nonlinear Theory and Applications, Las Vegas, Vol. 2, pp. 717-722.

Lotz, K. , Bölöni, L., Roska, T. \& Hámori, J. [1999] "Hyperacuity in time: A CNN model of a time-coding pathway of sound localization," IEEE Trans. Circuits Syst.-I 46, 994-1002.

Marr, D. [1982] Vision (Freeman, San Francisco).

Reinagel, P., Godwin, D., Sherman S. M. \& Koch, C. [1999] "Encoding of visual information by LGN bursts," J Neurophysiol. 81, 2558-2569.

Roska, B. \& Werblin, F. S. [2001] "Vertical interactions across ten parallel stacked representations in mammalian retina," Nature 410, 583-587.

Roska, T. \& Chua, L. O. [1993a] "The CNN universal machine: An analogic array computer," IEEE Trans. Circuits Syst.-II 40, 163-173.
Roska, T., Hámori, J., Lábos, E., Lotz, K., Orzó, L., Takács, J., Venetiáner, P. L., Vidnyánszky, Z. \& Zarándy, Á. [1993b] "The use of CNN models in the subcortical visual pathway," IEEE Trans. Circuits Syst.-I 40, 182-195.

Roska, T., Zarándy, Á. \& Chua, L. O. [1993c] "Color image processing by CNN," in Circuit Theory and Design. Proc. ECCTD'93, ed. Didiev. H (Elsevier, Amsterdam), pp. 57-62.

Sillito, A. M. \& Murphy, P. C. [1988] "GABAergic processes in the central visual system," in Neurotransmitters and Cortical Functions, eds. Dykes, R. W. \& Gloor, P. (Plenum Publ. Co.), pp. 167-185.

Takahashi, T. T. \& Konishi, M. [1986] "Selectivity for interaural time difference in the owl's midbrain," $J$. Neurosci. 6, 3413-3422.

Teeters, J. L. \& Werblin, F. S. [1991] "Real-time simulation of the retina allowing visualization of each processing stage," Image Understanding and the ManMachine Interface III., SPIE 1472, 6-17.

Wagner, H., Takahashi, T. \& Konishi, M. [1987] "Representation of interaural time difference in the central nucleus of the barn owl's inferior colliculus," J. Neurosci. 7, 3105-3116.

Werblin, F. S. [1991] "Synaptic connections, receptive fields and pattern of activity in the tiger salamander retina," Invest. Ophthalmol. Vis. Sci. 32, 459-483.

Werblin, F. S. \& Roska, B. [2004] "Multichannel retinal processing: A tutorial of retinal function from photoreceptors to parallel feature abstractions mediated by the analog array processing capabilities of the retina," Int. J. Bifurcation and Chaos 14, 843-852.

Zarándy, Á., Werblin, F., Roska, T. \& Chua L. O. [1994] "Novel types of analogic CNN algorithms for recognizing bank-notes," Proc. 3rd IEEE Int. Workshop on Cellular Neural Networks and their Applications (CNNA-94), Rome, Italy, pp. 273-278.

Zarándy Á., Orzó L., Grawes E. \& F. Werblin [1999] "CNN based early vision models for color vision and visual illusions," IEEE Trans. CAS-I: Special Issue on Bio-Inspired Processors and Cellular Neural Networks for Vision 46, 229-238. 
Copyright of International Journal of Bifurcation \& Chaos in Applied Sciences \& Engineering is the property of World Scientific Publishing Company and its content may not be copied or emailed to multiple sites or posted to a listserv without the copyright holder's express written permission. However, users may print, download, or email articles for individual use. 